\title{
Normal Forms for Three-dimensional Parametric Instabilities in Ideal Hydrodynamics
}

\author{
Edgar Knobloch \\ Department of Physics \\ University of California, Berkeley, CA 94720, USA \\ Alex Mahalov * \\ Department of Mathematics \\ Arizona State University, Tempe, AZ 85287 \\ Jerrold E. Marsden ${ }^{\dagger}$ \\ Department of Mathematics \\ University of California, Berkeley, CA 94720, USA
}

October, 1992. This version: December 20, 1993

\begin{abstract}
We derive and analyze several low dimensional Hamiltonian normal forms describing system symmetry breaking in ideal hydrodynamics. The equations depend on two parameters $(\epsilon, \lambda)$, where $\epsilon$ is the strength of a system symmetry breaking perturbation and $\lambda$ is a detuning parameter. In many cases the resulting equations are completely integrable and have an interesting Hamiltonian structure. Our work is motivated by three-dimensional instabilities of rotating columnar fluid flows with circular streamlines (such as the Burger vortex) subjected to precession, elliptical distortion or off-center displacement.
\end{abstract}

\section{Introduction}

In the last few years it has become recognized that elliptical distortion of a circular hydrodynamic flow can lead to instability (Pierrehumbert [1986], Bayly [1986], Vladimirov and Il'in [1988], Waleffe [1990]). The inclusion of precession can also lead to instability (Kerswell [1992], Mahalov [1993]). The unstable modes are characterized by an azimuthal wavenumber $m$, an axial wavenumber $k$ and a frequency $\omega$. Linear stability theory for Hamiltonian systems shows that an instability can only occur for wavenumbers and frequencies corresponding to intersections of dispersion curves for two distinct modes of oscillation or deformation of the circular flow, i.e., when $k_{1}=k_{2} \neq 0$ and $\omega_{1}=\omega_{2}$, with the latter perhaps both zero. Generically such a situation arises for a discrete set of axial wavenumbers. If the corresponding modes are coupled by distortion or precession, instability can result. For the elliptical distortion, this requires that the corresponding azimuthal wavenumbers differ by 2 , i.e., $m_{1}-m_{2}=2$. For the precessional instability the corresponding requirement is $m_{1}-m_{2}=1$. Such instabilities are of parametric type.

\footnotetext{
*Research partially supported by NSF Contract DMS 89-19074 and CTS 89-06343

${ }^{\dagger}$ Research partially supported by DOE Contract DE-FGO3-92ER25129.
} 
The above results have been obtained using linear stability theory. Guckenheimer and Mahalov [1992] observed that the presence of instability for eigenvalues near zero is intimately related to the reduction in symmetry by distortion or precession, and used this observation to describe the nonlinear evolution of the instability. In the present paper we extend this approach to eigenvalues away from zero, and examine in detail the effects of symmetry reduction on both the existence of instabilities and on their nonlinear evolution. We shall be mostly concerned with dynamical systems with the symmetry $S O(2) \times O(2)$, which arise frequently in applications. The origin of this symmetry varies widely, but the following serve as two prototypical examples:

Rotating columnar flows. A rotating columnar fluid flow with circular streamlines has $S O(2)$ symmetry with respect to rotations. If the system is, in addition, invariant under translations and reflections in the axial direction, the imposition of periodic boundary conditions introduces the symmetry $O(2)$.

Systems undergoing a Hopf bifurcation on a line. Many translation invariant hydrodynamical systems undergo a Hopf bifurcation at a particular parameter value. As above, the imposition of periodic boundary conditions together with reflection invariance introduces the spatial symmetry $O(2)$ into the dynamical equations. Normal form symmetry introduces an additional $S^{1} \cong S O(2)$ phase shift symmetry in time, leading to a Hopf bifurcation with $S O(2) \times O(2)$ symmetry.

Patterns result from spontaneous symmetry breaking instabilities that arise as parameters are varied, and these can be classified in terms of the isotropy subgroups of the full symmetry group. This spontaneous symmetry breaking must be distinguished from system symmetry breaking that arises due to symmetry breaking imperfections in the system. In applications it is essential to examine the robustness of the dynamical behavior of an idealized and usually highly symmetric model with respect to such imperfections. For example, for the rotating fluid column, such imperfections consist of distortions with various azimuthal wavenumbers, i.e., of various types of deformation of the circular streamlines. An additional source of imperfection is provided by precession of the column; see Mahalov [1993].

For systems undergoing a Hopf bifurcation, the imperfections are provided either by parametric forcing, breaking the $S^{1}$ phase shift symmetry, or by various types of spatial inhomogeneities (e.g., sidewalls) breaking the $O(2)$ spatial symmetry. Both types of imperfection are relevant to the Faraday system, in which surface waves are parametrically excited by the vertical oscillation of a fluid-filled container.

For the generic Hopf bifurcation with $S^{1} \times O(2)$ symmetry, the effects of breaking the $S^{1}$ symmetry down to $Z_{2}$ (2:1 resonance) was considered by Riecke et al. [1988]; the effect of breaking $O(2)$ down to $D_{2}$ was described in detail by Dangelmayr and Knobloch [1991]. Other aspects were considered by Nagata [1988].

In the present paper we discuss the role played by symmetry breaking imperfections in Hamiltonian systems. We show that imperfections can have dramatic consequences for the stability of the system, and discuss the requirements under which this is the case. There are three classes of problems that are of interest:

(a) the case of an eigenvalue $i \omega$ of double multiplicity in a system with $S O(2) \times S O(2)$ symmetry, corresponding to two distinct modes in the axial direction,

(b) the case of a pair of eigenvalues $\pm i \omega$ on the imaginary axis in a system with $S O(2) \times O(2)$ symmetry, and

(c) the case of double zero eigenvalue in a system with $S O(2) \times O(2)$ symmetry, corresponding to two steady modes related by reflection symmetry in the axial direction. 
Case (a) describes mode interactions characteristic of a system with $S O(2) \times S O(2)$ symmetry, such as might arise in the rotating fluid column with axial flow. Such interactions can be arranged by varying the axial wavenumber (i.e., the axial spatial period), and will be the primary focus of this paper. This is because the resulting amplitude or normal form equations subsume not only case (a) and a subset of case (b) but also the nondissipative limit of the normal form equations describing the Hopf bifurcation with $O(2)$ symmetry and the double Hopf bifurcation with 1:1 resonance with or without $S O(2)$ symmetry.

Two items of caution are in order here. Firstly, the number of eigenvalues participating in the Hamiltonian limit of, say, the Faraday problem, is double that in the dissipative case. Nonetheless, the formal nondissipative limit of the dissipative amplitude equations is of invaluable assistance in analyzing their dynamics, much as the nonlinear Schrödinger limit of the complex GinzburgLandau equation helps in understanding the dynamics of the latter. Note that Hamiltonian mode interactions in a system with $S O(2) \times O(2)$ symmetry (case (b) above) lead to more complicated equations on $\mathbf{C}^{4}$.

A second caution concerns the justification of the use of low dimensional Hamiltonian normal forms. For dissipative systems, one usually justifies such a procedure by the use of a center manifold (or sometimes an inertial manifold) because this manifold captures, in a precise sense, all of the local qualitative features of the dynamics in a low dimensional system. In the Hamiltonian case, this sort of procedure is not easy to justify rigorously, however. One approach is to imagine that the Hamiltonian system is embedded in a dissipative family and that after the dissipation is added along with bifurcation parameters, there will be a well defined low dimensional center manifold of fixed dimension independent of the small but nonzero dissipation. It would be naive to imagine that this center manifold along with its qualitative dynamics literally converges as the dissipation tends to zero, but one can nonetheless expect - and prove in various special instances (e.g., for equilibria or relative equilibria) - that many of the main features of the dissipative dynamics (sometimes representing the large scale dynamics) are well represented by the Hamiltonian system on the center manifold or an approximation to it. An instance where one can prove that the Hamiltonian part of the normal form captures some of the essential features of the general dynamics can be found in Lewis and Marsden [1989]. However, the general theory along these lines remains incomplete, although the theory of Mielke [1992] should prove to be very useful in this respect.

The movement of eigenvalues in generic, nonsymmetric Hamiltonian systems was described by Krein [1950] and Galin [1982]. Simple eigenvalues always remain on the imaginary axis under small Hamiltonian perturbations of any kind. When eigenvalues collide resulting in multiple eigenvalues lying on the imaginary axis, they may either split, leaving the imaginary axis, or pass through one another, remaining on the imaginary axis. In the former case the system becomes unstable. The movement of eigenvalues in generic families of symmetric Hamiltonian systems is affected by the symmetry type (see Dellnitz et al. [1992]). In particular the symmetry may force the eigenvalues to pass through one another and for many systems with symmetry, this can be the generic behavior. In this case the reduction in symmetry due to an imperfection may result in splitting "windows", and hence lead to instabilities (Guckenheimer and Mahalov [1992]). The resulting normal forms comprise a two-parameter family $(\epsilon, \lambda)$ of Hamiltonian vector fields. Here $\epsilon$ denotes the size of system symmetry breaking perturbation, while $\lambda$ is a detuning parameter, i.e., the difference in frequencies of the two interacting modes. Both are assumed to be small. In the following we derive the normal forms corresponding to a number of problems of this type, elucidate their Hamiltonian structure, and describe the resulting phase portraits and transitions in the $(\epsilon, \lambda)$ plane.

In $\S 2$ we describe the analysis relevant to understanding the instability of a precessing rotating fluid column. In $\S 3$ we discuss the elliptical instability. These cases are distinguished by the azimuthal wavenumber $m$ of the imperfection. The general case of parametric instabilities in systems with symmetry is discussed in $\S 4$. $\S 5$ is devoted to the Faraday system and the Benjamin-Feir insta- 
bility. In $\S 6$ we apply our ideas to rotating columnar fluid flows with circular streamlines undergoing slow precession. In the two-dimensional case, the energy-Casimir method can be used to prove stability by constructing a positive definite functional on a neighborhood of the basic flow (Szeri and Holmes [1988]). This functional becomes indefinite if a third dimension is added, allowing an instability by the reduced symmetry mechanism. The paper concludes with a discussion in $\S 7$.

\section{Hamiltonian normal forms: the case $m=1$}

\subsection{Basic amplitude equations}

In this section we consider Hamiltonian systems with $S O(2) \times S O(2)$ symmetry, such as the Euler equations for swirling fluid flow in a cylinder subject to periodic boundary conditions in the axial direction. We suppose that the system possesses a trivial (i.e., $S O(2) \times S O(2)$ invariant) equilibrium. A flow with circular streamlines and uniform in the axial direction serves as an important example. We are interested in the stability of this flow with respect to perturbations that break both azimuthal and axial invariance, i.e., with respect to three-dimensional perturbations. It is well known that the trivial state is stable with respect to a single mode of this type, and this continues to be the case when the axial wavelength is so chosen that two such modes have identical frequencies. However, if the symmetry of the system is broken so that these two modes couple, then instability becomes possible. We consider first the interaction between two modes both with eigenvalues $i \omega$, one of which is axisymmetric with the other having azimuthal wavenumber $m=1$. These are the modes that appear to dominate the process of vortex breakdown (see Leibovich et al. [1986] for a discussion). If $A_{1}$ and $A_{2}$ denote the complex amplitudes of these modes, the corresponding linear eigenfunction takes the form

$$
\psi(r, \phi, z)=\operatorname{Re}\left(A_{1} e^{i k z}+A_{2} e^{i k z+i \phi}\right) .
$$

Here, Re denotes the real part and $k$ is the axial wavenumber of both modes. The symmetry under translations $z \mapsto z+d$ in the axial direction acts by $\left(A_{1}, A_{2}\right) \mapsto e^{i k d}\left(A_{1}, A_{2}\right)$, while rotations $\phi \mapsto \phi+\theta$ act by $\left(A_{1}, A_{2}\right) \mapsto\left(A_{1}, A_{2} e^{i \theta}\right)$. These operations may be combined to yield the symmetry $\left(A_{1}, A_{2}\right) \mapsto\left(A_{1} e^{i \theta_{1}}, A_{2} e^{i \theta_{2}}\right)$. The resulting equivariant amplitude equations take the form

$$
\begin{aligned}
& \frac{d A_{1}}{d t}=i \omega A_{1}+i A_{1}\left(s_{11}\left|A_{1}\right|^{2}+s_{12}\left|A_{2}\right|^{2}\right)+\text { h.o.t. } \\
& \frac{d A_{2}}{d t}=i \omega A_{2}+i A_{2}\left(s_{21}\left|A_{1}\right|^{2}+s_{22}\left|A_{2}\right|^{2}\right)+\text { h.o.t., }
\end{aligned}
$$

where the $s_{i j}$ are real constants and h.o.t. denotes higher order terms. Since these equations are invariant under the symmetry $\left(A_{1}, A_{2}\right) \mapsto e^{i \omega \tau}\left(A_{1}, A_{2}\right)$ corresponding to phase shifts in time, they are already in normal form. In other problems considered below, the normal form transformations will introduce a distinct $S^{1}$ symmetry into the corresponding normal form.

When the symmetry with respect to rotations is reduced by system symmetry breaking with azimuthal wavenumber $m=1$, e.g., by precession or off-center displacement, the $S O(2)$ rotation symmetry is broken to the identity and the dominant symmetry breaking terms enter at linear order:

$$
\begin{aligned}
& \frac{d A_{1}}{d t}=i \omega A_{1}+\epsilon p A_{2}+i A_{1}\left(s_{11}\left|A_{1}\right|^{2}+s_{12}\left|A_{2}\right|^{2}\right)+\text { h.o.t. } \\
& \frac{d A_{2}}{d t}=i \omega A_{2}+\epsilon q A_{1}+i A_{2}\left(s_{21}\left|A_{1}\right|^{2}+s_{22}\left|A_{2}\right|^{2}\right)+\text { h.o.t. }
\end{aligned}
$$


Here, $\epsilon$ is a measure of the departure from full symmetry (e.g., $\epsilon$ could be the strength of an external Coriolis force), and is positive. All other symmetry breaking terms that can be added have to be at least cubic in the amplitudes and so are small relative to those retained. This approach is in the spirit of the corresponding dissipative analysis by Dangelmayr and Knobloch [1991]. In the following we drop the higher order terms.

The resulting equations are invariant under the operation $\left(A_{1}, A_{2}\right) \mapsto\left(e^{i \theta} A_{1}, e^{i \theta} A_{2}\right)$ only, i.e., under the diagonal action of $S O(2)$. In general we are interested in the situation where the two modes are not exactly in resonance. Consequently we replace equations (2.3) by

$$
\begin{aligned}
& \frac{d A_{1}}{d t}=i \omega_{1} A_{1}+\epsilon p A_{2}+i A_{1}\left(s_{11}\left|A_{1}\right|^{2}+s_{12}\left|A_{2}\right|^{2}\right)+\text { h.o.t. } \\
& \frac{d A_{2}}{d t}=i \omega_{2} A_{2}+\epsilon q A_{1}+i A_{2}\left(s_{21}\left|A_{1}\right|^{2}+s_{22}\left|A_{2}\right|^{2}\right)+\text { h.o.t. }
\end{aligned}
$$

where $\omega_{1}-\omega_{2} \equiv \lambda$ is the detuning. Then at $\lambda=0, \omega_{1}=\omega_{2}=\omega$. Finally, by going into the rotating frame $\left(A_{1}, A_{2}\right) \mapsto e^{i \omega_{2} t}\left(A_{1}, A_{2}\right)$, the equations can be further simplified:

$$
\begin{aligned}
& \frac{d A_{1}}{d t}=i \lambda A_{1}+\epsilon p A_{2}+i A_{1}\left(s_{11}\left|A_{1}\right|^{2}+s_{12}\left|A_{2}\right|^{2}\right)+\text { h.o.t. } \\
& \frac{d A_{2}}{d t}=\epsilon q A_{1}+i A_{2}\left(s_{21}\left|A_{1}\right|^{2}+s_{22}\left|A_{2}\right|^{2}\right)+\text { h.o.t. }
\end{aligned}
$$

Such time dependent transformations must be remembered when interpreting periodic (or other special) solutions of (2.5) in terms of the original system. Note that by rescaling $A_{1}$ and $A_{2}$ and redefining $\epsilon$ we may set $p=q=1$ (if $p q>1$ ) or $p=-q=1$ (if $p q<1$ ). The distinction between these two cases will be of vital importance in what follows. Identical equations hold in the more general case involving the interaction of two modes with azimuthal wavenumbers $m$ and $m+1$.

Equations (2.5) are usually written using $\bar{A}_{2}$ instead of $A_{2}$ as the variable (see below). In the dissipative case with the additional reflection symmetry $A_{1} \leftrightarrow A_{2}$, the resulting equations describe the effect of breaking the translation symmetry in a Hopf bifurcation with $O(2)$ symmetry. A detailed discussion of this case is given by Dangelmayr and Knobloch [1991]. In this case the coupling coefficient $p$ is in general complex, with $q=\bar{p}$, and the dynamics of the resulting equations depend sensitively on $\arg p$.

\subsection{Hamiltonian structure}

In the following we examine the Hamiltonian structure of equations (2.4), dropping the higher order terms. These equations can be written in the following two standard forms, depending on the sign of $p q$. First, if $p q>0$, we set $z_{1}=i A_{1}$ and $z_{2}=\bar{A}_{2}$ to get the $1:-1$ resonance form

$$
\begin{aligned}
& \dot{z}_{1}=i \omega_{1} z_{1}+i \epsilon p \bar{z}_{2}+i z_{1}\left(s_{11}\left|z_{1}\right|^{2}+s_{12}\left|z_{2}\right|^{2}\right) \\
& \dot{z}_{2}=-i \omega_{2} z_{2}+i \epsilon q \bar{z}_{1}-i z_{2}\left(s_{21}\left|z_{1}\right|^{2}+s_{22}\left|z_{2}\right|^{2}\right) .
\end{aligned}
$$

Second, if $p q<0$, we let $\zeta_{1}=z_{1}$ and $\zeta_{2}=\bar{z}_{2}$ to get the $1: 1$ resonance form

$$
\begin{aligned}
& \dot{\zeta}_{1}=i \omega_{1} \zeta_{1}+i \epsilon p \zeta_{2}+i \zeta_{1}\left(s_{11}\left|\zeta_{1}\right|^{2}+s_{12}\left|\zeta_{2}\right|^{2}\right) \\
& \dot{\zeta}_{2}=i \omega_{2} \zeta_{2}-i \epsilon q \zeta_{1}+i \zeta_{2}\left(s_{21}\left|\zeta_{1}\right|^{2}+s_{22}\left|\zeta_{2}\right|^{2}\right) .
\end{aligned}
$$


As already mentioned, we can assume that $p=q=1$ in (2.6) and that $p=-q=1$ in (2.7). We shall see that the former case corresponds to the splitting case, while the latter corresponds to the passing case.

We now consider the Hamiltonian nature of these systems. The Hamiltonian structure we use is the standard one obtained by taking the real and imaginary parts of $z_{i}$ and $\zeta_{i}$ as conjugate variables. For example, we write $z_{1}=q_{1}+i p_{1}$ and require $\dot{q}_{1}=\partial H / \partial p_{1}$ and $\dot{p}_{1}=-\partial H / \partial q_{1}$. A useful trick in this regard that enables one to work in complex notation is to write Hamilton's equations as $\dot{z}_{k}=-2 i \partial H / \partial \bar{z}_{k}$. Using this, one readily finds that:

(i) The system (2.6) is Hamiltonian if and only if $s_{12}=-s_{21}$ and $p=q$. In this case we can choose

$$
H\left(z_{1}, z_{2}\right)=\frac{1}{2}\left(\omega_{2}\left|z_{2}\right|^{2}-\omega_{1}\left|z_{1}\right|^{2}\right)-\epsilon p \operatorname{Re}\left(z_{1} z_{2}\right)-\frac{s_{11}}{4}\left|z_{1}\right|^{4}-\frac{s_{12}}{2}\left|z_{1} z_{2}\right|^{2}+\frac{s_{22}}{4}\left|z_{2}\right|^{4} .
$$

(ii) The system (2.7) is Hamiltonian if and only if $s_{12}=s_{21}$ and $p=-q$. In this case we can choose

$$
H\left(\zeta_{1}, \zeta_{2}\right)=-\frac{1}{2}\left(\omega_{1}\left|\zeta_{1}\right|^{2}+\omega_{2}\left|\zeta_{2}\right|^{2}\right)-\epsilon p \operatorname{Re}\left(\zeta_{1} \bar{\zeta}_{2}\right)-\frac{s_{11}}{4}\left|\zeta_{1}\right|^{4}-\frac{s_{12}}{2}\left|\zeta_{1} \zeta_{2}\right|^{2}-\frac{s_{22}}{4}\left|\zeta_{2}\right|^{4} .
$$

Note that for (2.6) with $\epsilon=0$ there are two separate $S^{1}$ actions acting on $z_{1}$ and $z_{2}$ independently; the corresponding conserved quantities are $\left|z_{1}\right|^{2}$ and $\left|z_{2}\right|^{2}$. However, for $\epsilon \neq 0$, the symmetry action is

$$
\left(z_{1}, z_{2}\right) \mapsto\left(e^{i \theta} z_{1}, e^{-i \theta} z_{2}\right)
$$

with the conserved quantity

$$
J\left(z_{1}, z_{2}\right)=\frac{1}{2}\left(\left|z_{1}\right|^{2}-\left|z_{2}\right|^{2}\right)
$$

Likewise, for (2.7), the symmetry action is

$$
\left(\zeta_{1}, \zeta_{2}\right) \mapsto\left(e^{i \theta} \zeta_{1}, e^{i \theta} \zeta_{2}\right)
$$

leading to the conserved quantity

$$
J\left(\zeta_{1}, \zeta_{2}\right)=\frac{1}{2}\left(\left|\zeta_{1}\right|^{2}+\left|\zeta_{2}\right|^{2}\right)
$$

In either case, it is clear that (2.6) and (2.7) are completely integrable systems, with the integrals being provided by the Hamiltonians and the corresponding conserved $J$. In view of the conservation of $J$, the Hamiltonians (2.8) and (2.9) can be further simplified. We choose not to do this in order to emphasize that the splitting and passing cases are distinguished by the structure of the corresponding Hamiltonians even in the absence of additional symmetries. Close to the origin (2.8) is indefinite (splitting), while (2.9) is definite (passing). In fact, the eigenvalues $\mu$ of the linearization of both at the origin in $\mathbf{C}^{2}$ are given by

$$
\mu=\frac{1}{2} i\left\{ \pm\left(\omega_{1}+\omega_{2}\right) \pm \sqrt{\lambda^{2}-4 \epsilon^{2} p q}\right\},
$$

where $\lambda=\omega_{1}-\omega_{2}$ is the detuning. In particular, if $p q>0$, one gets splitting along the lines $\lambda=2 \epsilon \sqrt{p q}$, in agreement with the generic theory of Dellnitz, Melbourne, and Marsden [1992]. (In the context of this theory, it is the symmetry $\left(z_{1}, z_{2}\right) \mapsto\left(z_{1}, e^{i \theta} z_{2}\right)$ that is broken, and the 
representations on the two spaces, which reduce to the eigenspaces at $\epsilon=0$, are distinct). In contrast, when $p q<0$ all four eigenvalues remain imaginary. However, in this case the passing of eigenvalues for $\epsilon=0$ changes to "bouncing" of eigenvalues for $\epsilon$ different from zero. Again this is consistent with the generic theory: when symmetry, other than normal form symmetry, is lost, passing is not generic. We expect that in the independent passing case the loss of symmetry always leads to splitting and a window of instability if the quadratic terms at criticality (here $\epsilon=0, \omega_{1}=\omega_{2}$ ) are indefinite, but that it leads to bouncing if they are definite).

Although the system (2.4) is Hamiltonian only in the special case identified above, it is nonetheless completely integrable. To see this we introduce the variables $r_{1}, r_{2}$ and $\phi \equiv \phi_{2}-\phi_{1}$, where $A_{1}=$ $r_{1} \exp \left(i \phi_{1}\right)$ and $A_{2}=r_{2} \exp \left(i \phi_{2}\right)$. The truncated system (2.5) then takes the form

$$
\begin{aligned}
\frac{d r_{1}}{d t} & =\epsilon p r_{2} \cos \phi \\
\frac{d r_{2}}{d t} & =\epsilon q r_{1} \cos \phi \\
\frac{d \phi}{d t} & =-\lambda-\frac{\epsilon}{r_{1} r_{2}}\left(q r_{1}^{2}+p r_{2}^{2}\right) \sin \phi+a r_{1}^{2}+b r_{2}^{2},
\end{aligned}
$$

where $a=s_{21}-s_{11}, b=s_{22}-s_{12}$. Observe that, without changing the values of $a$ and $b$, and hence without altering the preceding equations, we can adjust the constants in the original system so that it is Hamiltonian. In other words, the original system and consequently system (2.14) can be assumed to be Hamiltonian without any loss of generality. This system has the following two integrals obtained from the original momentum and the energy:

$$
J=\frac{1}{2 q}\left(q r_{1}^{2}-p r_{2}^{2}\right), \quad E=\frac{\lambda}{2\left(p^{2}+q^{2}\right)}\left(p r_{1}^{2}+q r_{2}^{2}\right)+\epsilon r_{1} r_{2} \sin \phi-\frac{a}{4 p} r_{1}^{4}-\frac{b}{4 q} r_{2}^{4} .
$$

Using these integrals, one can obtain a single differential equation for $\rho \equiv r_{1}^{2}$. This equation takes the form

$$
\left(\frac{d \rho}{d t}\right)^{2}=P(\rho)
$$

where

$$
P(\rho) \equiv 4 \epsilon^{2} p q \rho(\rho-2 J)-4 p^{2}\left(E+\frac{\lambda q^{2}}{p\left(p^{2}+q^{2}\right)} J-\frac{\lambda}{2 p} \rho+\frac{a}{4 p} \rho^{2}+\frac{b q}{4 p^{2}}(\rho-2 J)^{2}\right)^{2}
$$

is a polynomial of degree four. Clearly, $-P(\rho)$ may be thought of as the potential energy.

Some additional structure can be derived naturally by the method of Hamiltonian reduction (cf. Marsden [1992]) as follows. Let $\phi=\frac{\pi}{2}-\theta_{1}-\theta_{2}$, where $z_{1}=r_{1} \exp \left(i \theta_{1}\right), z_{2}=r_{2} \exp \left(i \theta_{2}\right)$. We know that the Hamiltonian structure for $(2.6)$ on $\mathbf{C}^{2}$ described above induces one on $\mathbf{C}^{2} / S^{1}$ and that the two integrals descend to the quotient space, as does the Poisson bracket. The quotient space $\mathbf{C}^{2} / S^{1}$ is parametrized by $\left(r_{1}, r_{2}, \phi\right)$ and dropping the integrals from the previous subsection reproduces (2.15). But one can also drop the Poisson bracket. That is, the equations in $\left(r_{1}, r_{2}, \phi\right)$ can be cast in Hamiltonian form $\dot{F}=\{F, H\}$ for the induced Poisson bracket. Here $H$ is given by (2.8). This bracket is obtained simply by using the chain rule to relate the complex variables and the polar coordinates. One finds that

$$
\{F, K\}\left(r_{1}, r_{2}, \phi\right)=-\frac{1}{r_{1}}\left(\frac{\partial F}{\partial r_{1}} \frac{\partial K}{\partial \phi}-\frac{\partial F}{\partial \phi} \frac{\partial K}{\partial r_{1}}\right)-\frac{1}{r_{2}}\left(\frac{\partial F}{\partial r_{2}} \frac{\partial K}{\partial \phi}-\frac{\partial F}{\partial \phi} \frac{\partial K}{\partial r_{2}}\right) .
$$

A similar procedure works for (2.7) with $\phi=\theta_{2}-\theta_{1}-\frac{\pi}{2}$, where $\zeta_{1}=r_{1} \exp \left(i \theta_{1}\right)$ and $\zeta_{2}=r_{2} \exp \left(i \theta_{2}\right)$. 
The Poisson bracket (2.18) is, of course, nothing but the original canonical Poisson bracket on the space of $\left(q_{1}, p_{1}\right)$ and $\left(q_{2}, p_{2}\right)$ variables, but written in the new polar coordinate variables, and as such, is an example of a noncanonical bracket. In the next section, we shall see other ways of writing the original canonical bracket in new variables. Such brackets are thus related to each other through their relation to the original canonical variables.

\subsection{Rigid body form of the amplitude equations}

In this section, we show that equations (2.3) or (2.5) can be written in rigid body form. This form is of independent interest, but one of the motivations for including it is the fact that the identification of invariant spheres and Euler type variables has often proved useful in bifurcation theory (Swift 1988).

We first recall some general theory for this situation by giving a version of the theory of Kummer [1979]. Consider the action of $S^{1}$ on $\mathbf{C}^{2}$ given by

$$
\left(z_{1}, z_{2}\right) \mapsto\left(e^{i k \theta} z_{1}, e^{i l \theta} z_{2}\right),
$$

where $k$ and $l$ are integers. This action is Hamiltonian with respect to the symplectic form on $\mathbf{C}^{2}$ given by

$$
\Omega\left(\left(z_{1}, z_{2}\right),\left(\tilde{z}_{1}, \tilde{z}_{2}\right)\right)=-\operatorname{Im}\left(z_{1} \overline{\tilde{z}}_{1}\right)-\operatorname{Im}\left(z_{2} \overline{\tilde{z}}_{2}\right) .
$$

The conserved quantity (or momentum map) for this action is given by

$$
J\left(z_{1}, z_{2}\right)=\frac{1}{2}\left(k\left|z_{1}\right|^{2}+l\left|z_{2}\right|^{2}\right) .
$$

The momentum map $J$ is invariant under the $S^{1}$ action. Other invariant functions are given by

$$
X+i Y=\bar{z}_{1}^{l} z_{2}^{k}, \quad Z=\frac{1}{2}\left(k\left|z_{1}\right|^{2}-l\left|z_{2}\right|^{2}\right) .
$$

If, say, $l$, is negative, then we replace $\bar{z}_{1}^{l}$ by $z_{1}^{|l|}$ in these expressions. Note that $-J \leq Z \leq J$ and that these invariants are related by

$$
X^{2}+Y^{2}=k^{-|l|} l^{-|k|}(J+Z)^{|l|}(J-Z)^{|k|} .
$$

In the case of the $1: 1$ resonance $(k=1, l=1)$ the invariants $(X, Y, Z)$ comprise the components of the momentum map of the standard $S U(2)$ action on $\mathbf{C}^{2}$; this action is relevant in this case, since it is the symmetry group of $J$.

For the $1: 1$ resonance with $J$ fixed equation (2.23) defines a sphere (Cushman and Rod [1982]). For the 1:-1 resonance $(k=1, l=-1$ ), one gets a hyperboloid (Iwai [1985]). For other values of $k$ and $l$ one can get objects with "pinches" and this is important in many resonance problems (see, for example, Haller and Wiggins [1992, 1993]).

In performing Poisson reduction, one normally constructs the quotient space $\mathbf{C}^{2} / S^{1}$ and calculates its induced Poisson bracket. However, except for the case of $k=1$ and $l=1$, the action, while locally free (apart from the origin), is not free, and so one has to be careful about singularities in the quotient space. For example, for $k=1$ and $l=2$, the action of the group element $e^{i \pi}$ leaves points of the form $\left(0, z_{2}\right) \in \mathbf{C}^{2}$ invariant. As we shall see shortly, the quotient in the Poisson context can be singularity free, even though the symplectic context has singularities.

For each real number $m$, define the map $\phi_{m}: \mathbf{R}^{3} \rightarrow \mathbf{R}$ by

$$
\phi_{m}=X^{2}+Y^{2}-k^{-|l|} l^{-|k|}(m+Z)^{|l|}(m-Z)^{|k|},
$$


so that the relation (2.23) between the variables $X, Y, Z, J$ becomes $\phi_{J}(X, Y, Z)=0$.

The quotient $\mathbf{C}^{2} / S^{1}$ is identifiable with $\mathbf{R}^{3}$ coordinatized by $(X, Y, Z)$ and carries the quotient Poisson structure given as follows. Let $F$ and $G$ be given functions of $X, Y, Z$ and let $(X, Y, Z)$ lie on the set $\phi_{m}(X, Y, Z)=0$. Then

$$
\{F, G\}(X, Y, Z)=\nabla \phi_{m} \cdot(\nabla F \times \nabla G) .
$$

This result is proved as follows. Define $f=F \circ \pi$ where $\pi$ is the map sending $\left(z_{1}, z_{2}\right) \mapsto(X, Y, Z)$. The Poisson bracket on $\mathbf{C}^{2}$ associated to the symplectic structure (2.20) is given by

$$
\{f, g\}=-\operatorname{Im}\left\langle\nabla_{z_{1}} f, \nabla_{z_{1}} g\right\rangle-\operatorname{Im}\left\langle\nabla_{z_{2}} f, \nabla_{z_{2}} g\right\rangle,
$$

where the gradient is the real gradient, taken with respect to the real inner product. The bracket $(2.25)$ follows on computing $\{f, g\}$ using the chain rule. This is a straightforward, although slightly lengthy computation.

The symplectic leaves in the above Poisson structure are given by the symplectic reduced spaces, namely by the sets $\phi_{m}=0$ corresponding to the symplectic reduced spaces $J^{-1}(m) / S^{1}$. The bracket above is the Poisson bracket associated with these leaves. The leaves $\phi_{m}=0 \subset \mathbf{R}^{3}$ are, in general, "pinched spheres". If $h$ is a Hamiltonian on $\mathbf{C}^{2}$ that is invariant under the action of $S^{1}$, it induces a function $H$ on $\mathbf{R}^{3}$ and the reduced equations on the pinched sphere $\phi_{m}=0$ are given by the (Euler-like) equations

$$
\dot{V}=\nabla H \times \nabla \phi_{m},
$$

where $V=(X, Y, Z)$. The case of multiple resonances is described by a similar expression, given by Kummer [1990].

The general theory just given shows that we should expect to get Euler-like equations when we express the equations (2.5) in terms of invariants, which is in fact borne out in what follows. Although the calculations below could be carried out in terms of the variables $z_{1}$ and $z_{2}$ used in equation (2.19) and the accompanying invariants (2.21), (2.22) we have chosen to employ the primitive variables used in equation (2.5). In particular, we use the invariants

$$
N=\left|A_{1}\right|^{2}+\left|A_{2}\right|^{2}, \quad u+i v=2 \bar{A}_{1} A_{2}, \quad w=\left|A_{1}\right|^{2}-\left|A_{2}\right|^{2},
$$

instead of $J, X, Y$ and $Z$ defined above, since these apply both when $p q>0$ and when $p q<0$. As before these invariants are not independent, since $N^{2}=u^{2}+v^{2}+w^{2}$. When $p=q=1$ (the splitting case) the quantity $w$ is an integral of the motion for equations (2.5), while the remaining quantities satisfy the top equation

$$
\frac{d L}{d t}=L \times \Omega
$$

where $L \equiv(N, i u, i v)^{T}$ and $\Omega \equiv P+D L$. Here $P$ is a vector and $D$ is a $3 \times 3$ matrix defined as follows:

$$
P=\left(\begin{array}{c}
\lambda-\frac{1}{2}\left(s_{12}+s_{21}-s_{11}-s_{22}\right) w \\
0 \\
-2 i \epsilon
\end{array}\right), \quad D=\left(\begin{array}{ccc}
-\frac{1}{2}\left(s_{21}-s_{12}+s_{22}-s_{11}\right) & 0 & 0 \\
0 & 0 & 0 \\
0 & 0 & 0
\end{array}\right)
$$

Specifically,

$$
\frac{d N}{d t}=2 \epsilon u, \quad \frac{d u}{d t}=v \Omega_{1}(w, N)+2 \epsilon N, \quad \frac{d v}{d t}=-u \Omega_{1}(w, N),
$$


where

$$
\Omega_{1}(w, N) \equiv \lambda-\frac{1}{2}\left(s_{12}+s_{21}-s_{11}-s_{22}\right) w-\frac{1}{2}\left(s_{21}-s_{12}+s_{22}-s_{11}\right) N .
$$

The kinetic energy can now be constructed as $T=L^{T} P+\frac{1}{2} L^{T} D L$, and is an integral of motion for the top $(d T / d t=0)$. Note that $T=4 E$, where $E$ is defined by $(2.15)$.

In the case $p=-q=1, N$ is an integral of (2.5), while

$$
\frac{d w}{d t}=2 \epsilon u, \quad \frac{d u}{d t}=-2 \epsilon w+v \Omega_{1}(w, N), \quad \frac{d v}{d t}=-u \Omega_{1}(w, N) .
$$

These equations can also be written in the form (2.29) with $L \equiv(w, u, v)^{T}$ and $\Omega \equiv P+D L$, where $P$ and $D$ are now defined as follows:

$$
P=\left(\begin{array}{c}
\lambda-\frac{1}{2}\left(s_{21}-s_{12}+s_{22}-s_{11}\right) N \\
0 \\
2 \epsilon
\end{array}\right), \quad D=\left(\begin{array}{ccc}
-\frac{1}{2}\left(s_{12}+s_{21}-s_{11}-s_{22}\right) & 0 & 0 \\
0 & 0 & 0 \\
0 & 0 & 0
\end{array}\right)
$$

\subsection{The simplest normal form}

Equations (2.5) can be further simplified using the transformation (Jorgensen and Christiansen [1992])

$$
A_{1}(t)=B_{1}(t) \exp (i \psi(t)), \quad A_{2}(t)=B_{2}(t) \exp (i \psi(t)),
$$

where $\psi(t)=\int_{t_{0}}^{t}\left(s_{21}\left|B_{1}\right|^{2}+s_{12}\left|B_{2}\right|^{2}\right) d s$. This transformation does not change the invariants $(2.28)$. The variables $B_{1}(t)$ and $B_{2}(t)$ obey the following equations:

$$
\begin{aligned}
& \frac{d B_{1}}{d t}=i \lambda B_{1}+\epsilon p B_{2}+i\left(s_{11}-s_{21}\right) B_{1}\left|B_{1}\right|^{2} \\
& \frac{d B_{2}}{d t}=\epsilon q B_{1}+i\left(s_{22}-s_{12}\right) B_{2}\left|B_{2}\right|^{2} .
\end{aligned}
$$

By rescaling the amplitudes $B_{1}, B_{2}$ and the time three constants in (2.36) can be eliminated. Redefining $\lambda$ and $\epsilon$ we obtain

$$
\begin{aligned}
& \frac{d B_{1}}{d t}=i \lambda B_{1}+\epsilon B_{2}+i B_{1}\left|B_{1}\right|^{2} \\
& \frac{d B_{2}}{d t}= \pm \epsilon B_{1}+i c B_{2}\left|B_{2}\right|^{2}
\end{aligned}
$$

where $c:= \pm\left(\frac{s_{22}-s_{12}}{s_{11}-s_{21}}\right) \frac{q}{p}$ is a real constant and $\lambda$ replaces $\lambda( \pm p q)^{-1 / 2}$. Equations (2.37) constitute our desired normal form, subject to the nondegeneracy conditions $s_{22} \neq s_{12}, s_{11} \neq s_{21}$, in addition to $p \neq 0, q \neq 0$.

In the + case (splitting) these equations have two conserved quantities $w$ and $H$, given by

$$
\begin{aligned}
w & =\left|B_{1}\right|^{2}-\left|B_{2}\right|^{2} \\
H(u, N) & =\frac{\lambda}{2} N+\frac{1-c}{8} N^{2}+\frac{1+c}{4} N w \pm \epsilon \sqrt{N^{2}-u^{2}-w^{2}} .
\end{aligned}
$$


Here $w, u$ and $N$ are defined as in (2.28). The two signs now refer to the two sheets of the hyperboloid; the results below are given for the + sign. Note that $H$ is a Hamiltonian for (2.37), and corresponds to the $1:-1$ resonance, as described above. In the variables $u$ and $N$, the equations take the form

$$
\begin{aligned}
\frac{d u}{d t} & =2 \sqrt{N^{2}-u^{2}-w^{2}} \frac{\partial H}{\partial N}=\{H, u\} \\
\frac{d N}{d t} & =-2 \sqrt{N^{2}-u^{2}-w^{2}} \frac{\partial H}{\partial u}=\{H, N\} .
\end{aligned}
$$

Here $\{$,$\} is the non-canonical Poisson bracket$

$$
\{f, g\} \equiv 2 \sqrt{N^{2}-u^{2}-w^{2}}\left(\frac{\partial f}{\partial N} \frac{\partial g}{\partial u}-\frac{\partial f}{\partial u} \frac{\partial g}{\partial N}\right) .
$$

These equations are equivalent to (2.31). The bracket (2.40) is the Lie-Poisson bracket for the hyperboloid (orbits of $S O(2,1)$ ).

In the - case (passing) equations (2.37) have instead the two integrals $N$ and $H$ given by

$$
\begin{aligned}
N & =\left|B_{1}\right|^{2}+\left|B_{2}\right|^{2} \\
H(u, w) & =\frac{\lambda}{2} w+\frac{1+c}{8} w^{2}+\frac{1-c}{4} N w+\epsilon \sqrt{N^{2}-u^{2}-w^{2}} .
\end{aligned}
$$

In the variables $u$ and $w$, the equations take the form

$$
\begin{aligned}
& \frac{d u}{d t}=2 \sqrt{N^{2}-u^{2}-w^{2}} \frac{\partial H}{\partial w}=\{H, u\} \\
& \frac{d w}{d t}=-2 \sqrt{N^{2}-u^{2}-w^{2}} \frac{\partial H}{\partial u}=\{H, w\},
\end{aligned}
$$

where

$$
\{f, g\} \equiv 2 \sqrt{N^{2}-u^{2}-w^{2}}\left(\frac{\partial f}{\partial w} \frac{\partial g}{\partial u}-\frac{\partial f}{\partial u} \frac{\partial g}{\partial w}\right)
$$

\subsection{Phase portraits for a two-parameter family $(\epsilon, \lambda)$ of Hamiltonian vec- tor fields in the + case (splitting)}

In this section we analyze the two-parameter family of Hamiltonian vector fields

$$
\begin{aligned}
\frac{d B_{1}}{d t} & =i \lambda B_{1}+\epsilon B_{2}+i B_{1}\left|B_{1}\right|^{2} \\
\frac{d B_{2}}{d t} & =\epsilon B_{1}+i c B_{2}\left|B_{2}\right|^{2} .
\end{aligned}
$$

FFrom (2.38) we have

$$
\epsilon v=H-\frac{\lambda}{2} N-\frac{1-c}{8} N^{2}-\frac{1+c}{4} N w
$$


so that

$$
\begin{aligned}
\frac{d N}{d t} & =2 \tilde{u} \\
\frac{d \tilde{u}}{d t} & =g_{0}+g_{1} N+g_{2} N^{2}+g_{3} N^{3}
\end{aligned}
$$

where $\tilde{u}=\epsilon u$ and

$$
\begin{aligned}
g_{0} & =\tilde{\lambda} H \\
g_{1} & =\frac{1}{2}\left(4 \epsilon^{2}-\tilde{\lambda}^{2}\right)+\frac{1-c}{2} H \\
g_{2} & =-\frac{3}{8}(1-c) \tilde{\lambda} \\
g_{3} & =-\frac{(1-c)^{2}}{16} .
\end{aligned}
$$

Here $\tilde{\lambda} \equiv \lambda+\frac{1}{2}(1+c) w$. Phase portraits of (2.46) depend on the number of real roots of the cubic polynomial $f(N) \equiv g_{0}+g_{1} N+g_{2} N^{2}+g_{3} N^{3}$. Let $P=g_{1} g_{3}-\frac{1}{3} g_{2}^{2}$ and $Q=g_{0} g_{3}^{2}-\frac{1}{3} g_{1} g_{2} g_{3}+\frac{2}{27} g_{2}^{3}$. If $\frac{Q^{2}}{4}+\frac{P^{3}}{27}>0$ then $f(N)$ has a single real root and the phase portrait is topologically equivalent to a circle. In the case $\frac{Q^{2}}{4}+\frac{P^{3}}{27}<0$, the polynomial $f(N)$ has three real roots and the phase portrait of (2.46) is topologically equivalent to a figure eight. Thus, the expression $\frac{Q^{2}}{4}+\frac{P^{3}}{27}$ can be used in determining phase portraits for a particular choice of parameters $\tilde{\lambda}, \epsilon, c$ and $H$.

The invariant surface $H=0$ is of particular interest. Restricted to this surface, equations (2.44) become

$$
\begin{aligned}
\frac{d N}{d t} & =2 \tilde{u} \\
\frac{d \tilde{u}}{d t} & =\frac{1}{2}\left(4 \epsilon^{2}-\tilde{\lambda}^{2}\right) N-\frac{3}{8}(1-c) \tilde{\lambda} N^{2}-\frac{(1-c)^{2}}{16} N^{3},
\end{aligned}
$$

These equations have three families of equilibria, given by $(\tilde{u}, N)=(0,0)$ and

$$
\left(0, \frac{3 \tilde{\lambda} \pm \sqrt{\tilde{\lambda}^{2}+32 \epsilon^{2}}}{c-1}\right),
$$

provided $N>0$. Of these, $(0,0)$ corresponds to the original equilibrium $\left(A_{1}, A_{2}\right)=(0,0)$ provided $w=0$. This is because $|w|<N$. This equilibrium is unstable in the wedge $|\lambda|<2 \epsilon$. The nontrivial equilibria depend on the value of the integral $w$. When $c>1$ there is no nontrivial equilibrium in $\tilde{\lambda}<-2 \epsilon$; in $-2 \epsilon<\tilde{\lambda}<2 \epsilon$ there is precisely one such equilibrium and it is stable. Finally, in $\tilde{\lambda}>2 \epsilon$ there are two nontrivial equilibria, with the larger one stable and the smaller one unstable. In the invariant surface $H=w=0$ the original equilibrium is stable for $\lambda<-2 \epsilon$; it loses stability to a new equilibrium as $\lambda$ passes through $\lambda=-2 \epsilon$. This equilibrium remains stable with increasing $\lambda$ even when $\lambda$ exceeds $2 \epsilon$, and the original equilibrium regains its stability. Thus for $\lambda>2 \epsilon$ the two stable equilibria coexist, and the original equilibrium is unstable with respect to finite amplitude perturbations. The situation is reversed when $c<1$; in this case the finite amplitude instabilities occur for $\lambda<-2 \epsilon$. The corresponding bifurcation diagram is shown in Figure 1. 


\subsection{Phase portraits for a two-parameter family $(\epsilon, \lambda)$ of Hamiltonian vec- tor fields in the - case (passing)}

In this section we analyze the two-parameter family of Hamiltonian vector fields

$$
\begin{aligned}
& \frac{d B_{1}}{d t}=i \lambda B_{1}+\epsilon B_{2}+i B_{1}\left|B_{1}\right|^{2} \\
& \frac{d B_{2}}{d t}=-\epsilon B_{1}+i c B_{2}\left|B_{2}\right|^{2} .
\end{aligned}
$$

The normal form (2.49) corresponds to the passing case. These equations may be written in the form

$$
\begin{aligned}
& \frac{d w}{d t}=2 \tilde{u} \\
& \frac{d \tilde{u}}{d t}=h_{0}+h_{1} w+h_{2} w^{2}+h_{3} w^{3}
\end{aligned}
$$

where $\tilde{u}=\epsilon u$ and

$$
\begin{aligned}
& h_{0}=\left(\lambda+\frac{1-c}{2} N\right) H \\
& h_{1}=-2 \epsilon^{2}-\frac{\lambda^{2}}{2}-\frac{1-c}{2} \lambda N-\frac{(1-c)^{2}}{8} N^{2}+\frac{1+c}{2} H \\
& h_{2}=-\frac{3}{8}(1+c)\left(\lambda+\frac{1-c}{2} N\right) \\
& h_{3}=-\frac{(1+c)^{2}}{16} .
\end{aligned}
$$

As before the invariant surface $H=0$ is of particular interest. There are three fixed points in this surface, given by $(\tilde{u}, w)=(0,0)$ and

$$
\left(0, \frac{-3 \tilde{\lambda} \pm \sqrt{\tilde{\lambda}^{2}-32 \epsilon^{2}}}{1+c}\right)
$$

where $\tilde{\lambda} \equiv \lambda+\frac{1}{2}(1+c) N$. Note that the nontrivial fixed points are present for $|\tilde{\lambda}|>4 \sqrt{2} \epsilon$. The trivial equilibrium corresponding to the original equilibrium is always stable, with $N$ serving locally as a Liapunov function. Of the remaining equilibria it is the one with the larger $|w|$ that is stable. Thus for $|\tilde{\lambda}|>4 \sqrt{2} \epsilon$ finite amplitude instabilities become possible, provided that these are associated with sufficiently large perturbations in $N$. The corresponding bifurcation diagram is shown in Figure 2 .

\subsection{The transition between splitting and passing}

The connection between the splitting and passing cases is simple to establish. We keep $p=1$ and allow $q$ to vary through zero. In this case, the two integrals are

$$
w \equiv q\left|B_{1}\right|^{2}-\left|B_{2}\right|^{2}
$$


and

$$
H \equiv \frac{\lambda N}{1+q^{2}}+\epsilon v+\frac{1}{2} \frac{N}{\left(1+q^{2}\right)^{2}}[(1-c q) N+2 w(c+q)],
$$

where $N \equiv\left|B_{1}\right|^{2}+q\left|B_{2}\right|^{2}$ and $(u, v)$ are defined as before. The structure of the problem remains of the form (2.46), with

$$
\begin{aligned}
& g_{0}=\tilde{\lambda} H-2 \epsilon^{2}\left(\frac{1-q^{2}}{1+q^{2}}\right) w \\
& g_{1}=\frac{1}{1+q^{2}}\left[H+4 \epsilon^{2} q-\tilde{\lambda}^{2}\right] \\
& g_{2}=-\frac{3}{2} \frac{1-c q}{\left(1+q^{2}\right)^{2}} \tilde{\lambda} \\
& g_{3}=-\frac{1}{2} \frac{(1-c q)^{2}}{\left(1+q^{2}\right)^{3}},
\end{aligned}
$$

where

$$
\tilde{\lambda} \equiv \lambda+\frac{c+q}{1+q^{2}} w
$$

In the surface $\{H=0\} \cap\{w=0\}$ containing the origin the dynamical equations simplify:

$$
\begin{aligned}
\frac{d \tilde{N}}{d t} & =2 \tilde{u} \\
\frac{d \tilde{u}}{d t} & =\frac{1}{2}\left(4 \epsilon^{2} q-\lambda^{2}\right) \tilde{N}-\frac{3}{8} \lambda \tilde{N}^{2}-\frac{1}{16} \tilde{N}^{3},
\end{aligned}
$$

where

$$
(\tilde{N}, \tilde{u}) \equiv(1-c q)\left(\frac{2 N}{1+q^{2}}, \epsilon u\right),
$$

Note that the small $q$ regime is equivalent to the case of small $c$. One can now see that for fixed $(\epsilon, \lambda)$ as $q$ decreases towards zero the instability region closes up, the two bifurcation points $\lambda= \pm 2 \epsilon \sqrt{q}$ come together and the branch of nontrivial equilibria detaches from the trivial equilibrium $\tilde{N}=0$. Indeed one can verify that $d \tilde{N} / d \lambda=0, \infty$ both require $q<0$. The transition between Figure $1 \mathrm{~b}$ and Figure 2 thus takes place via the degenerate bifurcation diagrams shown in Figure 3.

\subsection{Mode interaction with $S O(2) \times O(2)$ symmetry}

We conclude this section by writing down the corresponding normal forms for the case with reflection symmetry in the axial direction. Then the symmetry group associated with translations and reflections in the axial direction becomes $O(2)$, and the full symmetry is now $S O(2) \times O(2)$. In this case the modes at $\pm i \omega$ are related by reflection symmetry. Let $A_{3}$ and $A_{4}$ be the amplitudes of the modes at $-i \omega$ defined so that the linear eigenfunction is

$$
\psi(r, \phi, z)=\operatorname{Re}\left(A_{1} e^{i k z}+A_{2} e^{i k z+i \phi}+\bar{A}_{3} e^{-i k z}+\bar{A}_{4} e^{-i k z+i \phi}\right) .
$$

The translations $z \rightarrow z+d$ now act by $\left(A_{1}, A_{2}, A_{3}, A_{4}\right) \rightarrow e^{i k d}\left(A_{1}, A_{2}, A_{3}, A_{4}\right)$, while the reflection $z \rightarrow-z$ acts by $\left(A_{1}, A_{2}, A_{3}, A_{4}\right) \rightarrow\left(\bar{A}_{3}, \bar{A}_{4}, \bar{A}_{1}, \bar{A}_{2}\right)$. Finally, the rotations $\phi \rightarrow \phi+\theta$ act 
by $\left(A_{1}, A_{2}, A_{3}, A_{4}\right) \rightarrow\left(A_{1}, e^{i \theta} A_{2}, A_{3}, e^{-i \theta} A_{4}\right)$. In addition, in normal form, the vector field will commute with the normal form symmetry $\left(A_{1}, A_{2}, A_{3}, A_{4}\right) \rightarrow\left(e^{i \omega \tau} A_{1}, e^{i \omega \tau} A_{2}, e^{-i \omega \tau} A_{3}, e^{-i \omega \tau} A_{4}\right)$ generated by phase shifts $t \rightarrow t+\tau$. The most general Hamiltonian vector field commuting with these operations, truncated at third order, is (cf. Silber and Knobloch [1991], Knobloch and Silber [1993])

$$
\begin{gathered}
\frac{d A_{1}}{d t}=i \omega A_{1}+i A_{1}\left(s_{11}\left|A_{1}\right|^{2}+s_{12}\left|A_{2}\right|^{2}+s_{13}\left|A_{3}\right|^{2}+s_{14}\left|A_{4}\right|^{2}\right)+i r_{1} A_{2} A_{4} \bar{A}_{3} \\
\frac{d A_{2}}{d t}=i \omega A_{2}+i A_{2}\left(s_{21}\left|A_{1}\right|^{2}+s_{22}\left|A_{2}\right|^{2}+s_{23}\left|A_{3}\right|^{2}+s_{24}\left|A_{4}\right|^{2}\right)+i r_{2} A_{1} A_{3} \bar{A}_{4} \\
\frac{d A_{3}}{d t}=-i \omega A_{3}-i A_{3}\left(s_{13}\left|A_{1}\right|^{2}+s_{14}\left|A_{2}\right|^{2}+s_{11}\left|A_{3}\right|^{2}+s_{12}\left|A_{4}\right|^{2}\right)-i r_{1} A_{2} A_{4} \bar{A}_{1} \\
\frac{d A_{4}}{d t}=-i \omega A_{4}-i A_{4}\left(s_{23}\left|A_{1}\right|^{2}+s_{24}\left|A_{2}\right|^{2}+s_{21}\left|A_{3}\right|^{2}+s_{22}\left|A_{4}\right|^{2}\right)-i r_{2} A_{1} A_{3} \bar{A}_{2} .
\end{gathered}
$$

With detuning and the symmetry breaking terms due to precession or off-center displacement we obtain

$$
\begin{gathered}
\frac{d A_{1}}{d t}=i \omega_{1} A_{1}+\epsilon p A_{2}+i A_{1}\left(s_{11}\left|A_{1}\right|^{2}+s_{12}\left|A_{2}\right|^{2}+s_{13}\left|A_{3}\right|^{2}+s_{14}\left|A_{4}\right|^{2}\right)+i r_{1} A_{2} A_{4} \bar{A}_{3} \\
\frac{d A_{2}}{d t}=i \omega_{2} A_{2}+\epsilon q A_{1}+i A_{2}\left(s_{21}\left|A_{1}\right|^{2}+s_{22}\left|A_{2}\right|^{2}+s_{23}\left|A_{3}\right|^{2}+s_{24}\left|A_{4}\right|^{2}\right)+i r_{2} A_{1} A_{3} \bar{A}_{4} \\
\frac{d A_{3}}{d t}=-i \omega_{1} A_{3}+\epsilon p A_{4}-i A_{3}\left(s_{13}\left|A_{1}\right|^{2}+s_{14}\left|A_{2}\right|^{2}+s_{11}\left|A_{3}\right|^{2}+s_{12}\left|A_{4}\right|^{2}\right)-i r_{1} A_{2} A_{4} \bar{A}_{1} \\
\frac{d A_{4}}{d t}=-i \omega_{2} A_{4}+\epsilon q A_{3}-i A_{4}\left(s_{23}\left|A_{1}\right|^{2}+s_{24}\left|A_{2}\right|^{2}+s_{21}\left|A_{3}\right|^{2}+s_{22}\left|A_{4}\right|^{2}\right)-i r_{2} A_{1} A_{3} \bar{A}_{2} .
\end{gathered}
$$

In this form the equations commute with the $O(2)$ axial symmetry, as well as with the normal form symmetry; only the $S O(2)$ rotation symmetry has been broken. Identical equations hold for the corresponding interaction between modes with azimuthal wavenumbers $m$ and $m+1$.

Equations (2.58) generalize equations (2.4) to the case of $S O(2) \times O(2)$ symmetry. Note that the subspace $A_{3}=A_{4}=0$ is invariant. Within this subspace equations (2.58) reduce to (2.4), and describe the interaction between two downward propagating waves. Another important invariant subspace is given by $A_{1}=\bar{A}_{3}, A_{2}=\bar{A}_{4}$. In this subspace equations (2.58) also take the form (2.4), but with different coefficients. The equations within this subspace describe the interaction between two standing waves. The general equations describe the interaction between traveling and standing waves of both types $(m=0, m=1)$. In particular the equations capture the stability properties of downward traveling waves with respect to upward traveling waves, or the stability properties of standing waves with respect to traveling perturbations.

The Hamiltonian structure of the equations (2.58) may be analyzed in the same manner as in \$2.2. For example, if $p q>0$, and $z_{1}=i A_{1}, z_{2}=A_{2}, z_{3}=i A_{3}, z_{4}=A_{4}$ we see that the equations are Hamiltonian if $p=q=1, s_{12}=-s_{21}$, and $r_{1}=r_{2}$. As before, these conditions are inessential in the sense that when one reduces to polar coordinates, one can always arrange for them to be satisfied. In addition, there is a Hamiltonian structure for the equations written in terms of invariants.

In the absence of system symmetry breaking the dissipative version of equations (2.57) was analyzed (in particular cases) by Silber and Knobloch [1991] and by Knobloch and Silber [1993]. 
With the system symmetry breaking terms the corresponding equations in the $A_{3}=A_{4}=0$ subspace form the nondissipative limit of the equations studied by Dangelmayr and Knobloch [1991] for the Hopf bifurcation with $O(2)$ symmetry. In particular, the nontrivial steady states described in $\S 2.4$ and $\S 2.5$ now correspond to the two types of standing waves described in their paper. This is also the case for the equations in the $A_{1}=\bar{A}_{3}, A_{2}=\bar{A}_{4}$ subspace. The corresponding analysis for the competition of two modes, as in the present case, will be reported elsewhere.

\section{Hamiltonian normal forms: the case $m=2$ (elliptical in- stability)}

\subsection{Basic amplitude equations}

We next consider the case in which the azimuthal wavenumbers differ by 2 . This is the case of the so-called elliptic instability studied by Pierrehumbert [1986] and Bayly [1986]. It arises when the rotation symmetry $S O(2)$ is broken to $Z_{2}$, for example, by distorting the circular streamlines of the flow into elliptical shape. This distortion couples modes propagating in the positive and negative directions along the axis. In systems with axial reflection symmetry both waves are simultaneously present. For the eigenfunction

$$
\psi(r, \phi, z)=\operatorname{Re}\left(A_{1} e^{i k z+i \phi}+A_{2} e^{-i k z+i \phi}\right),
$$

the rotations $\phi \rightarrow \phi+\theta$ act by $\left(A_{1}, A_{2}\right) \rightarrow e^{i \theta}\left(A_{1}, A_{2}\right)$, while the translations $z \rightarrow z+d$ act by $\left(A_{1}, A_{2}\right) \rightarrow\left(e^{i k d} A_{1}, e^{-i k d} A_{2}\right)$ and reflection $z \rightarrow-z$ acts by $\left(A_{1}, A_{2}\right) \rightarrow\left(A_{2}, A_{1}\right)$. As a consequence of the reflection symmetry the dispersion curves cross on the real axis, i.e., at $\omega=0$. The elliptical distortion breaks the rotation invariance and couples the two counter-propagating modes. The amplitude equations, truncated at third order, take the form

$$
\begin{gathered}
\frac{d A_{1}}{d t}=i \lambda A_{1}+\epsilon \bar{A}_{2}+i A_{1}\left(s_{1}\left|A_{1}\right|^{2}+s_{2}\left|A_{2}\right|^{2}\right) \\
\frac{d A_{2}}{d t}=i \lambda A_{2}+\epsilon \bar{A}_{1}+i A_{2}\left(s_{2}\left|A_{1}\right|^{2}+s_{1}\left|A_{2}\right|^{2}\right),
\end{gathered}
$$

where, as before, $\lambda$ is the detuning and $\epsilon$ measures the size of the elliptical distortion. The coefficient describing the coupling to the distortion can be made purely real as in (3.2), or purely imaginary. In either case the origin is unstable in the wedge $|\lambda|<\epsilon$, i.e.,, the reflection symmetry $z \rightarrow-z$ forces splitting to take place. This observation is independent of any detailed considerations of the system of interest. In the dissipative case equations (3.2) were studied by Riecke et al. [1988] (see also Walgraef [1988]), in the context of parametric forcing of the Hopf bifurcation with $O(2)$ symmetry. Riecke et al. showed that the forcing stabilized standing waves over traveling waves, even in cases where, in the absence of forcing, traveling waves would be stable. Equations (3.2) thus represent the nondissipative limit of the analysis of Riecke et al.

With the change of variables $A_{1}(t)=B_{1}(t) \exp (i \psi(t)), A_{2}(t)=B_{2}(t) \exp (-i \psi(t))$, where $\psi(t)=$ $-s_{2} \int_{t_{0}}^{t}\left(\left|B_{1}\right|^{2}-\left|B_{2}\right|^{2}\right) d s$, one obtains

$$
\begin{aligned}
& \frac{d B_{1}}{d t}=i \lambda B_{1}+\epsilon \bar{B}_{2}+i\left(s_{1}+s_{2}\right) B_{1}\left|B_{1}\right|^{2} \\
& \frac{d B_{2}}{d t}=i \lambda B_{2}+\epsilon \bar{B}_{1}+i\left(s_{1}+s_{2}\right) B_{2}\left|B_{2}\right|^{2},
\end{aligned}
$$


or, rescaling $B_{1}$ and $B_{2}$ :

$$
\begin{gathered}
\frac{d B_{1}}{d t}=i \lambda B_{1}+\epsilon \bar{B}_{2}+i B_{1}\left|B_{1}\right|^{2} \\
\frac{d B_{2}}{d t}=i \lambda B_{2}+\epsilon \bar{B}_{1}+i B_{2}\left|B_{2}\right|^{2} .
\end{gathered}
$$

This is the required normal form for the elliptical instability, provided $s_{1}+s_{2} \neq 0$. Here $\lambda$ is the detuning parameter and $\epsilon$ measures the size of the elliptical distortion (e.g. eccentricity). It is remarkable that the normal form (3.4) does not contain any parameters depending on a specific problem under consideration.

Equations (3.4) are Hamiltonian in the standard structure for the complex variables $z_{1}=B_{1}$ and $z_{2}=B_{2}$ with the Hamiltonian

$$
H\left(B_{1}, B_{2}\right)=-\frac{\lambda}{2}\left(\left|B_{1}\right|^{2}+\left|B_{2}\right|^{2}\right)+\epsilon \operatorname{Im}\left(B_{1} B_{2}\right)-\frac{1}{4}\left(\left|B_{1}\right|^{4}+\left|B_{2}\right|^{4}\right) .
$$

Moreover, the symmetry $\left(B_{1}, B_{2}\right) \mapsto\left(e^{i \theta} B_{1}, e^{-i \theta} B_{2}\right)$ gives the conserved quantity (momentum map)

$$
J\left(B_{1}, B_{2}\right)=\left|B_{1}\right|^{2}-\left|B_{2}\right|^{2} .
$$

As in $\S 2$, we can express this Hamiltonian structure in either polar coordinates or in terms of invariants. In terms of the variables $B_{1} \equiv r_{1} \exp \left(i \phi_{1}\right), B_{2} \equiv r_{2} \exp \left(i \phi_{2}\right)$ and $\phi \equiv \phi_{1}+\phi_{2}$, equations (3.4) become

$$
\begin{aligned}
\frac{d r_{1}}{d t} & =\epsilon r_{2} \cos \phi \\
\frac{d r_{2}}{d t} & =\epsilon r_{1} \cos \phi \\
\frac{d \phi}{d t} & =2 \lambda-\epsilon\left(\frac{r_{2}}{r_{1}}+\frac{r_{1}}{r_{2}}\right) \sin \phi+r_{1}^{2}+r_{2}^{2} .
\end{aligned}
$$

Again, this system is completely integrable with the two integrals derived from the above Hamiltonian (or its negative) and momentum:

$$
J=r_{1}^{2}-r_{2}^{2}, \quad E=\frac{\lambda}{2}\left(r_{1}^{2}+r_{2}^{2}\right)-\epsilon r_{1} r_{2} \sin \phi+\frac{1}{4}\left(r_{1}^{4}+r_{2}^{4}\right) .
$$

Using the integrals $J$ and $E$, the solution of (3.7) reduces to quadrature:

$$
\frac{1}{4}\left(\frac{d \rho}{d t}\right)^{2}=P(\rho) \equiv \epsilon^{2}\left(\rho^{2}-J^{2}\right)-4\left(E-\frac{\lambda \rho}{2}-\frac{1}{8}\left(\rho^{2}+J^{2}\right)\right)^{2} .
$$

Here $\rho \equiv r_{1}^{2}+r_{2}^{2}$. Note that for fixed $J$ the integral $E$ varies between $h_{1}(J)$ and $h_{2}(J)$, where $h_{1}(J)$ corresponds to a fixed point and $h_{2}(J)$ corresponds to a homoclinic (heteroclinic) orbit.

The system (3.4) has two invariant subspaces $\left\{B_{1}=B_{2}\right\}$ and $\left\{B_{1}=i B_{2}\right\}$. In the dissipative case mentioned above these correspond to the two types of oscillations phase locked to half the frequency of the parametric forcing. Both are standing oscillations. These subspaces are characterized by $J=0$. In the first subspace (3.4) reduce to

$$
\frac{d B}{d t}=i \lambda B+\epsilon \bar{B}+i B|B|^{2}
$$


In the second subspace,

$$
\frac{d B}{d t}=i \lambda B-i \epsilon \bar{B}+i B|B|^{2} .
$$

The equations in each subspace are again Hamiltonian, which is consistent with the general fact that fixed point spaces of discrete symplectic symmetries are symplectic spaces and induce Hamiltonian subsystems on them (see Marsden [1992, Chapter 8] for the general theory). The corresponding phase portraits of (3.10) are shown in Figure 4. The phase portraits of (3.11) are obtained by rotation of the phase portraits in Figure 4 by $\frac{\pi}{2}$.

More generally, the phase portraits depend on both $E$ and $J$. These quantities specify invariant surfaces in phase space. Dynamics on these surfaces can be understood by converting (3.9) to a system of two equations in the variables $\rho$ and $\frac{d \rho}{d t}$. For $\lambda=0$ there are two possibilities, depending on the sign of $\epsilon^{2}+E-\frac{J^{2}}{8}$. The resulting phase portraits in $\left(\rho, \frac{d \rho}{d t}\right)$ space resemble those shown in Figure 4.

\subsection{The effect of symmetry breaking}

The discussion of the elliptical instability presented above relies on the presence of reflection symmetry in the axial direction. This residual symmetry need not be exact, however, and may be broken for example by means of an axial flow. A number of other system symmetry breaking perturbations can also be envisaged. These include the following:

(a) $S O(2) \times O(2) \rightarrow Z_{2} \times O(2) \rightarrow Z_{2} \times S O(2)$

(b) $S O(2) \times O(2) \rightarrow Z_{3} \times O(2)$

(c) $S O(2) \times O(2) \rightarrow Z_{4} \times O(2)$.

In those cases where the axial reflection symmetry is preserved, the elliptical instability remains a steady state one. In cases where it is broken (for example, by axial flow) the instability becomes a Hopf bifurcation (cf. Armbruster and Mahalov [1992], Knobloch [1992b]). In the following we suppose that $\epsilon_{1}$ measures the strength of the system symmetry breaking from $S O(2)$ to $Z_{n}$, and $\epsilon_{2}$ measures the strength of the system symmetry breaking from $O(2)$ to $S O(2)$, and retain as before only the dominant symmetry breaking terms.

When an axial flow reduces the symmetry from $S O(2) \times O(2)$ to $S O(2) \times S O(2)$ by breaking the reflection symmetry $z \rightarrow-z$ the symmetry $\left(B_{1}, B_{2}\right) \rightarrow\left(B_{2}, B_{1}\right)$ of the normal form (3.4) is broken. Consequently we can describe the effect of weak axial flow by breaking the latter symmetry. We obtain

$$
\begin{aligned}
& \frac{d B_{1}}{d t}=i \lambda_{1} B_{1}+\epsilon_{1} p_{1} \bar{B}_{2}+i\left(1+\gamma_{1}\right) B_{1}\left|B_{1}\right|^{2} \\
& \frac{d B_{2}}{d t}=i \lambda_{2} B_{2}+\epsilon_{1} p_{2} \bar{B}_{1}+i\left(1+\gamma_{2}\right) B_{2}\left|B_{2}\right|^{2} .
\end{aligned}
$$

Here, $\lambda_{2}-\lambda_{1}=O\left(\epsilon_{2}\right), p_{2}-p_{1}=O\left(\epsilon_{2}\right)$ and $\gamma_{2}-\gamma_{1}=O\left(\epsilon_{2}\right)$, where $\epsilon_{2}$ denotes the strength of the reflection symmetry breaking effect. Note that by rescaling the amplitudes the coefficients $\gamma_{1}$ and $\gamma_{2}$ can be set equal to zero, though at the cost of redefining $p_{1}$ and $p_{2}$. It is now easy to check that the trivial equilibrium is unstable whenever

$$
\left(\lambda_{1}-\lambda_{2}\right)^{2}<4\left(\epsilon_{1}^{2} p_{1} p_{2}-\lambda_{1} \lambda_{2}\right)
$$


Note that, in contrast to the symmetric case, the presence of the instability depends critically on the splitting of the frequencies of the left-handed and right-handed modes. If this splitting is large enough for a given value of $\epsilon$ the instability can be suppressed entirely.

Equations (3.12) are also integrable, and have the following two integrals:

$$
J=p_{2} r_{1}^{2}-p_{1} r_{2}^{2}, \quad E=\frac{1}{2}\left(\frac{\lambda_{1}+\lambda_{2}}{p_{1}+p_{2}}\right)\left(r_{1}^{2}+r_{2}^{2}\right)-\epsilon_{1} r_{1} r_{2} \sin \phi+\frac{1+\gamma_{1}}{4 p_{1}} r_{1}^{4}+\frac{1+\gamma_{2}}{4 p_{2}} r_{2}^{4} .
$$

The use of the integrals reduces the system (3.12) to quadrature:

$$
\frac{1}{4 p_{1} p_{2}}\left(\frac{d \rho}{d t}\right)^{2}=P(\rho)
$$

where $\rho \equiv p_{2} r_{1}^{2}+p_{1} r_{2}^{2}$ and

$$
\begin{aligned}
P(\rho) & \equiv \epsilon_{1}^{2}\left(\rho^{2}-J^{2}\right)-4 p_{1} p_{2}\left[E-\frac{\lambda_{1}+\lambda_{2}}{4 p_{1} p_{2}}\left(\rho+\frac{p_{1}-p_{2}}{p_{1}+p_{2}} J\right)\right. \\
& -\frac{1}{16 p_{1}^{2} p_{2}^{2}}\left[\left(\left(1+\gamma_{1}\right) p_{1}+\left(1+\gamma_{2}\right) p_{2}\right)\left(\rho^{2}+J^{2}\right)+2\left(\left(1+\gamma_{1}\right) p_{1}\right.\right. \\
& \left.\left.\left.-\left(1+\gamma_{2}\right) p_{2}\right) \rho J\right]\right]^{2} .
\end{aligned}
$$

Equations (3.4) and (3.12) can be put in rigid body form. We start with the equations

$$
\begin{aligned}
& \frac{d A_{1}}{d t}=i \lambda_{1} A_{1}+\epsilon_{1} p_{1} \bar{A}_{2}+i A_{1}\left(s_{11}\left|A_{1}\right|^{2}+s_{12}\left|A_{2}\right|^{2}\right) \\
& \frac{d A_{2}}{d t}=i \lambda_{2} A_{2}+\epsilon_{1} p_{2} \bar{A}_{1}+i A_{2}\left(s_{21}\left|A_{1}\right|^{2}+s_{22}\left|A_{2}\right|^{2}\right),
\end{aligned}
$$

describing the effect of weak axial flow on the elliptic instability (case (a) above). Here the amplitudes $A_{1}$ and $A_{2}$ are as in (3.1), and $\lambda_{1}-\lambda_{2}, p_{1}-p_{2}, s_{11}-s_{22}$ and $s_{12}-s_{21}$ are all $O\left(\epsilon_{2}\right)$. There are two cases: (i) $p_{1} p_{2}<0$ (pinched spheres, passing of the zero eigenvalues), and (ii) $p_{1} p_{2}>0$ (pinched hyperboloids, splitting of the zero eigenvalues). In case (ii), a rescaling of the amplitudes yields

$$
\begin{aligned}
& \frac{d A_{1}}{d t}=i \lambda_{1} A_{1}+\epsilon_{1} p \bar{A}_{2}+i A_{1}\left(s_{11}\left|A_{1}\right|^{2}+s_{12}\left|A_{2}\right|^{2}\right) \\
& \frac{d A_{2}}{d t}=i \lambda_{2} A_{2}+\epsilon_{1} p \bar{A}_{1}+i A_{2}\left(s_{21}\left|A_{1}\right|^{2}+s_{22}\left|A_{2}\right|^{2}\right) .
\end{aligned}
$$

In terms of the Euler variables

$$
N \equiv\left|A_{1}\right|^{2}+\left|A_{2}\right|^{2}, \quad w \equiv\left|A_{1}\right|^{2}-\left|A_{2}\right|^{2}, \quad u+i v=2 A_{1} A_{2},
$$

cf. equations (2.28), one obtains the equations

$$
\begin{aligned}
& \frac{d w}{d t}=0 \\
& \frac{d N}{d t}=2 \epsilon_{1} p u
\end{aligned}
$$




$$
\begin{aligned}
& \frac{d u}{d t}=-v\left(2 \lambda+a N+\epsilon_{2} b w\right)+2 \epsilon_{1} p N \\
& \frac{d v}{d t}=u\left(2 \lambda+a N+\epsilon_{2} b w\right),
\end{aligned}
$$

where $2 \lambda \equiv \lambda_{1}+\lambda_{2}, 2 a \equiv s_{11}+s_{21}+s_{12}+s_{22}$ and $2 \epsilon_{2} b \equiv s_{11}+s_{21}-s_{12}-s_{22}$. Note that once again $N^{2}=u^{2}+v^{2}+w^{2}$. If we replace $\epsilon_{1} p$ by $\epsilon_{1}$ and $\epsilon_{2} b$ by $\epsilon_{2}$ the vector $L \equiv(N, i u, i v)$ satisfies the top equation

$$
\frac{d L}{d t}=L \times \Omega
$$

where $\Omega \equiv P+D L$, and $P$ and $D$ are now given by

$$
P=\left(\begin{array}{c}
-2 \lambda-\epsilon_{2} w \\
0 \\
-2 i \epsilon_{1}
\end{array}\right), \quad D=\left(\begin{array}{ccc}
-a & 0 & 0 \\
0 & 0 & 0 \\
0 & 0 & 0
\end{array}\right) \text {, }
$$

cf. equation (2.30). As in the case of the $m=1$ instability, the kinetic energy is given by $T=$ $L^{T} P+\frac{1}{2} L^{T} D L$ and is an integral of the motion. It is now easy to understand how the Hamiltonian structure (and phase portraits) change when the two system symmetry breaking parameters $\epsilon_{1}$ and $\epsilon_{2}$ are varied.

To understand case (b) we note, following Nagata [1988], that the normal form for a vector field that commutes with the symmetry $Z_{3} \times O(2)$ is given by

$$
\begin{aligned}
& \frac{d A_{1}}{d t}=i g_{1} A_{1}+i \epsilon_{1} g_{2} \bar{A}_{1}^{2} \bar{A}_{2}^{3} \\
& \frac{d A_{2}}{d t}=i g_{3} A_{2}+i \epsilon_{1} g_{4} \bar{A}_{2}^{2} \bar{A}_{1}^{3},
\end{aligned}
$$

where the functions $g_{1}, \ldots, g_{4}$ are $C^{\infty}$ functions of the invariants $\left|A_{1}\right|^{2}+\left|A_{2}\right|^{2},\left(A_{1} A_{2}\right)^{3},\left(\bar{A}_{1} \bar{A}_{2}\right)^{3}$ and $\left(\left|A_{1}\right|^{2}-\left|A_{2}\right|^{2}\right)^{2}$. These functions are not independent since the two equations for $A_{1}$ and $A_{2}$ are related by the reflection symmetry $\left(A_{1}, A_{2}\right) \rightarrow\left(A_{2}, A_{1}\right)$.

Similarly, in case (c), one finds that the normal form that commutes with the required action of $Z_{4} \times O(2)$ is

$$
\begin{aligned}
& \frac{d A_{1}}{d t}=i g_{1} A_{1}+i \epsilon_{1} g_{2} \bar{A}_{1} \bar{A}_{2}^{2} \\
& \frac{d A_{2}}{d t}=i g_{3} A_{2}+i \epsilon_{1} g_{4} \bar{A}_{2} \bar{A}_{1}^{2}
\end{aligned}
$$

where the functions $g_{1}, \ldots, g_{4}$ are now functions of the invariants $\left|A_{1}\right|^{2}+\left|A_{2}\right|^{2},\left(A_{1} A_{2}\right)^{2},\left(\bar{A}_{1} \bar{A}_{2}\right)^{2}$ and $\left(\left|A_{1}\right|^{2}-\left|A_{2}\right|^{2}\right)^{2}$. As before these functions are related by the reflection symmetry $\left(A_{1}, A_{2}\right) \rightarrow$ $\left(A_{2}, A_{1}\right)$.

It is important to observe that the symmetry breaking terms that now enter are all nonlinear and hence they do not affect the linear stability problem. Consequently in these cases instability will not in general be present. This is because the wavenumbers of the eigenfunctions differ by 2 . However, when $S O(2)$ is broken to $Z_{2 n}(n \geq 1)$ the same equations as (3.18) are obtained for the 
interaction of modes of the form $e^{i k z+i n \phi}$ and $e^{-i k z+i n \phi}$. Similarly, when $m_{1}-m_{2}=2 n-1$, say, equations of the form (2.3) or (2.58) follow for modes of the form $e^{i k z}$ and $e^{i k z+i(2 n-1) \phi}$ whenever the symmetry is broken to $Z_{2 n-1}$, depending on the symmetry in the axial direction. Here $n$ is again a positive integer.

Equations (3.2) discussed above also arise in the theory of edge waves excited by a normally incident wave at a beach; equations (3.12) then describe the excitation of edge waves by slightly oblique waves (cf. Miles [1991]). This problem is closely related to that discussed in $\S 4$.

\section{The general case}

In this section we consider the interaction of two modes with azimuthal wavenumbers $m$ and $n$, and the same axial wavenumber. We must now distinguish between two types of parametric interaction, through coupling to a deformation mode of the form $e^{ \pm i(m-n) \phi}$ or of the form $e^{ \pm i(m+n) \phi}$, where $0<n<m$. We write the linear eigenfunction in the form

$$
\psi(r, \phi, z)=\operatorname{Re}\left(A_{m} e^{i k z+i m \phi}+A_{n} e^{i k z+i n \phi}+A_{-m} e^{i k z-i m \phi}+A_{-n} e^{i k z-i n \phi}\right) .
$$

The translations $z \rightarrow z+d$ now act by

$$
\left(A_{m}, A_{n}, A_{-m}, A_{-n}\right) \rightarrow e^{i k d}\left(A_{m}, A_{n}, A_{-m}, A_{-n}\right),
$$

while the reflection $z \rightarrow-z$ acts by

$$
\left(A_{m}, A_{n}, A_{-m}, A_{-n}\right) \rightarrow\left(\bar{A}_{-m}, \bar{A}_{-n}, \bar{A}_{m}, \bar{A}_{n}\right) .
$$

Finally, the rotations $\phi \rightarrow \phi+\theta$ act by

$$
\left(A_{m}, A_{n}, A_{-m}, A_{-n}\right) \rightarrow\left(e^{i m \theta} A_{m}, e^{i n \theta} A_{n}, e^{-i m \theta} A_{-m}, e^{-i n \theta} A_{-n}\right) .
$$

In addition, in normal form, the vector field will commute with the normal form symmetry

$$
\left(A_{m}, A_{n}, A_{-m}, A_{-n}\right) \rightarrow\left(e^{i \omega \tau} A_{m}, e^{i \omega \tau} A_{n}, e^{-i \omega \tau} A_{-m}, e^{-i \omega \tau} A_{-n}\right)
$$

generated by phase shifts $t \rightarrow t+\tau$. The most general Hamiltonian vector field commuting with these operations, truncated at third order, is

$$
\begin{aligned}
\frac{d A_{m}}{d t} & =i \omega A_{m}+i A_{m}\left(s_{11}\left|A_{m}\right|^{2}+s_{12}\left|A_{n}\right|^{2}+s_{13}\left|A_{-m}\right|^{2}+s_{14}\left|A_{-n}\right|^{2}\right)+i r_{1} A_{n} A_{-n} \bar{A}_{-m} \\
\frac{d A_{n}}{d t} & =i \omega A_{n}+i A_{n}\left(s_{21}\left|A_{m}\right|^{2}+s_{22}\left|A_{n}\right|^{2}+s_{23}\left|A_{-m}\right|^{2}+s_{24}\left|A_{-n}\right|^{2}\right)+i r_{2} A_{m} A_{-m} \bar{A}_{-n} \\
\frac{d A_{-m}}{d t} & =-i \omega A_{-m}-i A_{-m}\left(s_{13}\left|A_{m}\right|^{2}+s_{14}\left|A_{n}\right|^{2}+s_{11}\left|A_{-m}\right|^{2}+s_{12}\left|A_{-n}\right|^{2}\right)-i r_{1} A_{n} A_{-n} \bar{A}_{m} \\
\frac{d A_{-n}}{d t} & =-i \omega A_{-n}-i A_{-n}\left(s_{23}\left|A_{m}\right|^{2}+s_{24}\left|A_{n}\right|^{2}+s_{21}\left|A_{-m}\right|^{2}+s_{22}\left|A_{-n}\right|^{2}\right)-i r_{2} A_{m} A_{-m} \bar{A}_{n} .
\end{aligned}
$$

With detuning and the symmetry breaking terms of the form $e^{i(m-n) \phi}$ we now obtain

$$
\frac{d A_{m}}{d t}=i \omega_{1} A_{m}+i \epsilon p A_{n}+i A_{m}\left(s_{11}\left|A_{m}\right|^{2}+s_{12}\left|A_{n}\right|^{2}+s_{13}\left|A_{-m}\right|^{2}+s_{14}\left|A_{-n}\right|^{2}\right)+i r_{1} A_{n} A_{-n} \bar{A}_{-m}
$$




$$
\begin{aligned}
\frac{d A_{n}}{d t} & =i \omega_{2} A_{n}+i \epsilon q A_{m}+i A_{n}\left(s_{21}\left|A_{m}\right|^{2}+s_{22}\left|A_{n}\right|^{2}+s_{23}\left|A_{-m}\right|^{2}+s_{24}\left|A_{-n}\right|^{2}\right)+i r_{2} A_{m} A_{-m} \bar{A}_{-n} \\
\frac{d A_{-m}}{d t} & =-i \omega_{1} A_{-m}-i \epsilon p A_{-n}-i A_{-m}\left(s_{13}\left|A_{m}\right|^{2}+s_{14}\left|A_{n}\right|^{2}+s_{11}\left|A_{-m}\right|^{2}+s_{12}\left|A_{-n}\right|^{2}\right)-i r_{1} A_{n} A_{-n} \bar{A}_{m} \\
\frac{d A_{-n}}{d t} & =-i \omega_{2} A_{-n}-i \epsilon q A_{-m}-i A_{-n}\left(s_{23}\left|A_{m}\right|^{2}+s_{24}\left|A_{n}\right|^{2}+s_{21}\left|A_{-m}\right|^{2}+s_{22}\left|A_{-n}\right|^{2}\right)-i r_{2} A_{m} A_{-m} \bar{A}_{n} .
\end{aligned}
$$

The equations corresponding to the perturbation $e^{i(m+n) \phi}$ can be obtained from those above by replacing $n$ with $-n$. Note that the two sets of equations differ in the nonlinear terms, since the change of sign of $n$ is not in general a symmetry of the $S O(2) \times O(2)$ equivariant problem. The Hamiltonian structure of these equations is the same as that described in $\S 2.8$.

The corresponding equations for the $S O(2) \times S O(2)$ interaction can be obtained from those above by setting $A_{-m}$ and $A_{-n}$ equal to zero (cf. equations (2.3)). Moreover setting $A_{n}$ and $A_{-n}$ equal to zero results in equations of the form (3.2). Note that the structure of these generalizations does not differ from the special cases already considered. Consequently no further analysis is necessary. This is because of the translation invariance in the axial direction which prevents terms that are resonant in the azimuthal coordinate from appearing in the normal form equations. Note also that we have restricted attention in the above discussion to the interaction between modes with the same axial wavenumbers only. It is not hard to generalize the discussion to cases where the competing modes have different axial wavenumbers as well.

\section{Amplitude equations for parametrically driven capillary waves and Benjamin-Feir instability}

\subsection{Parametrically driven capillary waves}

As already mentioned the equations derived above for the elliptical instability are the same as those arising in parametrically forced systems. In this section we discuss in more detail one such example: Milner's [1991] model for secondary instabilities in driven capillary waves. Milner is interested in understanding the relative stability between surface waves with roll and square planforms in a shallow layer of water contained in a large aspect ratio container oscillated vertically. In this configuration the dominant restoring force is due to surface tension, and the resulting surface ripples are called capillary waves. In the following we assume translation symmetry in two orthogonal directions, as well as reflection symmetry with respect to both directions. We write the eigenfunction describing the elevation of the surface relative to the oscillating undeformed surface in the form

$$
\zeta(x, y, t)=\operatorname{Re}\left\{e^{i \omega t}\left(v_{1} e^{i k x}+v_{2} e^{i k y}+w_{1} e^{-i k x}+w_{2} e^{-i k y}\right)\right\} .
$$

The translation symmetry $(x, y) \rightarrow\left(x+d_{1}, y+d_{2}\right)$ acts by

$$
\left(v_{1}, v_{2}, w_{1}, w_{2}\right) \rightarrow\left(e^{i k d_{1}} v_{1}, e^{i k d_{2}} v_{2}, e^{-i k d_{1}} w_{1}, e^{-i k d_{2}} w_{2}\right) ;
$$

reflection $(x, y) \rightarrow(x,-y)$ acts by

$$
\left(v_{1}, v_{2}, w_{1}, w_{2}\right) \rightarrow\left(v_{1}, w_{2}, w_{1}, v_{2}\right),
$$

while rotation through $\pi / 2$ acts by

$$
\left(v_{1}, v_{2}, w_{1}, w_{2}\right) \rightarrow\left(w_{2}, v_{1}, v_{2}, w_{1}\right)
$$


Finally, the normal form symmetry acts by $\left(v_{1}, v_{2}, w_{1}, w_{2}\right) \rightarrow e^{i \phi}\left(v_{1}, v_{2}, w_{1}, w_{2}\right)$. The resulting normal form equations, truncated at third order, take the Hamiltonian form (Milner [1991], Silber and Knobloch [1991])

$$
\begin{aligned}
& \frac{d v_{1}}{d t}=i \lambda v_{1}+i \epsilon \bar{w}_{1}+i v_{1}\left(a\left|w_{1}\right|^{2}+b\left(\left|v_{1}\right|^{2}+\left|w_{1}\right|^{2}\right)+c\left(\left|v_{2}\right|^{2}+\left|w_{2}\right|^{2}\right)\right)+i d v_{2} w_{2} \bar{w}_{1} \\
& \frac{d v_{2}}{d t}=i \lambda v_{2}+i \epsilon \bar{w}_{2}+i v_{2}\left(a\left|w_{2}\right|^{2}+b\left(\left|v_{2}\right|^{2}+\left|w_{2}\right|^{2}\right)+c\left(\left|v_{1}\right|^{2}+\left|w_{1}\right|^{2}\right)\right)+i d v_{1} w_{1} \bar{w}_{2} \\
& \frac{d w_{1}}{d t}=i \lambda w_{1}+i \epsilon \bar{v}_{1}+i w_{1}\left(a\left|v_{1}\right|^{2}+b\left(\left|v_{1}\right|^{2}+\left|w_{1}\right|^{2}\right)+c\left(\left|v_{2}\right|^{2}+\left|w_{2}\right|^{2}\right)\right)+i d v_{2} w_{2} \bar{v}_{1} \\
& \frac{d w_{2}}{d t}=i \lambda w_{2}+i \epsilon \bar{v}_{2}+i w_{2}\left(a\left|v_{2}\right|^{2}+b\left(\left|v_{2}\right|^{2}+\left|w_{2}\right|^{2}\right)+c\left(\left|v_{1}\right|^{2}+\left|w_{1}\right|^{2}\right)\right)+i d v_{1} w_{1} \bar{v}_{2}
\end{aligned}
$$

cf. equation (2.57). Here $\epsilon$ measures the amplitude of the parametric forcing and is responsible for the breaking of the normal form symmetry $\left(v_{1}, v_{2}, w_{1}, w_{2}\right) \rightarrow e^{i \phi}\left(v_{1}, v_{2}, w_{1}, w_{2}\right)$. The parametric forcing respects the spatial symmetries. The quantity $\lambda$ is the frequency mismatch, $\lambda \equiv \omega-\frac{1}{2} \omega_{d}$, where $\omega_{d}$ is the forcing frequency. Here $\omega$ is the natural oscillation frequency of the capillary waves.

The equations in the invariant subspace $v_{2}=w_{2}=0$ take the form

$$
\begin{aligned}
\frac{d v_{1}}{d t} & =i \lambda v_{1}+i \epsilon \bar{w}_{1}+i v_{1}\left(a\left|w_{1}\right|^{2}+b\left(\left|v_{1}\right|^{2}+\left|w_{1}\right|^{2}\right)\right) \\
\frac{d w_{1}}{d t} & =i \lambda w_{1}+i \epsilon \bar{v}_{1}+i w_{1}\left(a\left|v_{1}\right|^{2}+b\left(\left|v_{1}\right|^{2}+\left|w_{1}\right|^{2}\right)\right) .
\end{aligned}
$$

In the dissipative case these are the equations studied by Riecke et al. [1988] and Walgraef [1988]; they describe the effect of parametric forcing on the competition between standing and traveling waves. There is another important invariant subspace of equations (5.2), given by $v_{1}=w_{1}, v_{2}=$ $w_{2}$. This subspace corresponds to standing waves in the two orthogonal directions. Consequently traveling wave perturbations are suppressed. In this subspace the equations take the form

$$
\begin{aligned}
& \frac{d v_{1}}{d t}=i \lambda v_{1}+i \epsilon \bar{v}_{1}+i v_{1}\left(a\left|v_{1}\right|^{2}+b\left|v_{2}\right|^{2}\right)+i d v_{2}^{2} \bar{v}_{1}, \\
& \frac{d v_{2}}{d t}=i \lambda v_{2}+i \epsilon \bar{v}_{2}+i v_{2}\left(b\left|v_{1}\right|^{2}+a\left|v_{2}\right|^{2}\right)+i d v_{1}^{2} \bar{v}_{2} .
\end{aligned}
$$

These equations describe the parametric resonance in small aspect ratio square containers and have been studied by a number of authors (e.g., Nagata [1989]). Nearly square containers may be studied by breaking weakly the symmetry $\left(v_{1}, v_{2}\right) \rightarrow\left(v_{2}, v_{1}\right)$ in equations (5.4) (cf. Feng and Sethna [1989, 1990]; Feng and Wiggins [1993]). In a rectangular container the required mode interaction problem is of codimension two since the frequencies of the two competing modes must be tuned in order to resonate with half the frequency of the parametric forcing. Such an interaction is described by the equations

$$
\begin{aligned}
& \frac{d v_{1}}{d t}=i \lambda_{1} v_{1}+i \epsilon p_{1} \bar{v}_{1}+i v_{1}\left(s_{11}\left|v_{1}\right|^{2}+s_{12}\left|v_{2}\right|^{2}\right)+i d_{1} v_{2}^{2} \bar{v}_{1} \\
& \frac{d v_{2}}{d t}=i \lambda_{2} v_{2}+i \epsilon p_{2} \bar{v}_{2}+i v_{2}\left(s_{21}\left|v_{1}\right|^{2}+s_{22}\left|v_{2}\right|^{2}\right)+i d_{2} v_{1}^{2} \bar{v}_{2}
\end{aligned}
$$


with $\lambda_{1}-\lambda_{2}=O(1)$ and similarly for the remaining coefficients. Note that no transformation of the type used to simplify equations (3.2) is available in this case. As in our earlier discussions, the above Hamiltonian equations have two sets of integrals corresponding to the energy and, when there is an $S^{1}$ symmetry present, a conserved momentum. In particular, the system (5.3) is completely integrable while (5.4) and (5.5) are completely integrable if $\epsilon=0$. For example, equations (5.5) with $\epsilon=0$, has the integrals

$$
J=d_{2} r_{1}^{2}+d_{1} r_{2}^{2}, \quad E=\frac{\lambda_{1}}{d_{1}} r_{1}^{2}+\frac{\lambda_{2}}{d_{2}} r_{2}^{2}+r_{1}^{2} r_{2}^{2} \cos \phi+\frac{s_{11}-s_{21}}{2 d_{1}} r_{1}^{4}+\frac{s_{22}-s_{12}}{2 d_{2}} r_{2}^{4},
$$

where we have used the polar coordinate notation $v_{1}=r_{1} e^{i \phi_{1}}, v_{2}=r_{2} e^{i \phi_{2}}$ and defined $\phi \equiv 2 \phi_{1}-2 \phi_{2}$. (As before, one adjusts the coefficients in (5.5) without changing the reduced system to get a standard Hamiltonian system). A special case of these integrals was found already by Feng and Sethna [1990]. The existence of these integrals should prove helpful in analyzing the parametrically forced problem (5.5) for $0<\epsilon \ll 1$, as in the work of Feng and Sethna [1990] and Feng and Wiggins [1993], as should those obtaining in the case $\epsilon \neq 0, d_{1}=d_{2}=0$, discussed in $\S 3$. We also remark that there are hidden symmetries in the above problems, particularly in the square case (Crawford [1992]). These are relevant in the Hamiltonian case since Neumann boundary conditions have to be imposed at the boundaries of the container.

\subsection{Benjamin-Feir instability}

In the example discussed above, the parametric instability arose in the standard way, i.e., by temporal modulation of a parameter of the system, in this case the gravitational acceleration. There is, however, another example of parametric instability in the theory of water waves that also fits into our picture. This is the so-called Benjamin-Feir instability of wavetrains. This is a modulational instability of the wavetrain and arises through the coupling of two sidebands, $k \pm l$, via the wavenumber $k$ of the wavetrain. Two cases are of interest, that in which the original wavetrain is a progressive wavetrain, and that in which the original wavetrain is a standing wave. These two cases differ by the presence of a reflection symmetry in vertical planes in the latter case. This instability may be viewed as follows: the undisturbed water surface plays the role of the basic state corresponding to the flow with circular streamlines. The wavetrain then provides the distortion that can couple two natural modes of oscillation of the system leading to the possibility of subharmonic instabilities of Benjamin-Feir type.

We illustrate the above discussion with the nonlinear Schrödinger equation

$$
\frac{\partial A}{\partial t}=i \gamma \frac{\partial^{2} A}{\partial x^{2}}+i|A|^{2} A
$$

subject to periodic boundary conditions in the spatial variable $x$. Equation (5.7) has a solution in the form of a wave $A=R e^{i \Omega t+i k x}$, where $\Omega=R^{2}-\gamma k^{2}$. We wish to study the stability of this solution with respect to side band perturbations, i.e., with respect to perturbations with wavenumbers $k \pm l$. Thus we set

$$
A=e^{i \Omega t}\left(R e^{i k x}+a\right)
$$

Linearizing in $a$, we find that $a$ satisfies the equation

$$
\frac{\partial a}{\partial t}=i \gamma \frac{\partial^{2} a}{\partial x^{2}}+i\left(2 R^{2}-\Omega\right) a+i R^{2} e^{2 i k x} \bar{a}
$$


This equation has a solution of the form

$$
a=b_{1} e^{i(k+l) x}+b_{2} e^{i(k-l) x},
$$

where

$$
\begin{aligned}
& \frac{d b_{1}}{d t}=i\left[2 R^{2}-\Omega-\gamma(k+l)^{2}\right] b_{1}+i R^{2} \bar{b}_{2} \\
& \frac{d b_{2}}{d t}=i\left[2 R^{2}-\Omega-\gamma(k-l)^{2}\right] b_{2}+i R^{2} \bar{b}_{1} .
\end{aligned}
$$

These equations are of the form (3.12), with $\lambda_{1}-\lambda_{2}=O(l), p_{1}=p_{2}=-1$, and $R^{2}$ playing the role of $\epsilon$. Condition (3.13) implies that an instability is present whenever

$$
0<\gamma\left(2 R^{2}-\gamma l^{2}\right)
$$

Consequently the wavetrain is unstable with respect to sideband instability $(0<l \ll 1)$ whenever $\gamma>0$. This is the Benjamin-Feir instability (cf. Benjamin [1967]).

In this discussion we have focused on the linear stability properties of a propagating wavetrain. To determine the nonlinear terms responsible for the saturation of the instability one would have to go through a center manifold type of reduction based on the two unstable modes. The structure of these terms is not as simple as in (3.12); this is because in the above derivation we are considering an instability of a wave, and this wave has already broken the $O(2)$ symmetry of the system. On the other hand if we consider the two sidebands, $k \pm l$, as two modes that are (weakly) coupled by a small amplitude wave, then to leading order (in $R$ ) the equations for $b_{1}$ and $b_{2}$ will be of the form

$$
\begin{aligned}
& \frac{d b_{1}}{d t}=i R^{2} \bar{b}_{2}+i g_{11} b_{1}+i g_{12} \bar{b}_{1}^{k-l-1} b_{2}^{k+l} \\
& \frac{d b_{2}}{d t}=i R^{2} \bar{b}_{1}+i g_{21} b_{2}+i g_{22} \bar{b}_{2}^{k+l-1} b_{1}^{k-l},
\end{aligned}
$$

where the functions $g_{i j}, i=1,2, j=1,2$, are functions of

$$
\left|b_{1}\right|^{2},\left|b_{2}\right|^{2}, b_{1}^{k-l} \bar{b}_{2}^{k+l}+\bar{b}_{1}^{k-l} b_{2}^{k+l} \text { and } \quad\left(b_{1}^{k-l} \bar{b}_{2}^{k+l}-\bar{b}_{1}^{k-l} b_{2}^{k+l}\right)^{2}
$$

as well as of $R$. For $k>2, l \geq 1, k>l$, the resulting equations truncated at third order are precisely of the form (3.2), though with broken reflection symmetry $\left(b_{1} \leftrightarrow b_{2}\right)$, and so can be transformed into equations (3.12). The Benjamin-Feir instability is thus of the same kind as the instabilities discussed here. We remark, finally, that the instability of standing wavetrains in dispersive systems is complicated by the finite group velocity of the waves, and so is described by equations that are more complicated than (5.7) (see Knobloch [1992a]).

\section{Precessional instability of columnar flows: an explicit ex- ample}

In this section we discuss an explicit application of the above ideas. We focus on the precessional instability of columnar flows of the form $(0, V(r), W(r))$, where $(r, \phi, z)$ are right-handed cylindrical coordinates. Szeri and Holmes [1988] have established sufficient conditions for the nonlinear stability of such flows to finite amplitude axisymmetric disturbances using the energy-Casimir method. The 
method depends upon finding a constant of motion that has a local maximum or minimum at the corresponding equilibrium. In general such a constant of motion is a functional of the kinetic energy and the conserved quantities that correspond to symmetries of the system via Noether's theorem. The nonlinear stability to axisymmetric perturbations is proved by showing that the second variation of this functional is positive (negative) definite. We show that the energy-Casimir functional becomes indefinite if three-dimensional variations are allowed. The idea of the proof is as follows. We assume that an infinitesimal external Coriolis force is applied to the system. As a result the system loses some of its conserved quantities (e.g. angular momentum about the $z$ axis). In addition the Coriolis force alters the base flow. We show that the resulting steady state flow has an unstable manifold for an arbitrarily small strength of the external Coriolis force, and conclude that columnar flows are structurally unstable in the sense that they are infinitesimally close to flows (steady state solutions of Euler equations) having an unstable manifold. We formalize this discussion by making the following definition: A steady-state solution $V_{0}$ of a Hamiltonian system with a Hamiltonian $H_{0}$ is called structurally unstable if for any $\epsilon_{0}>0$ there exists an $\epsilon, 0<\epsilon<\epsilon_{0}$, such that the steady state $V_{0}$ is deformed into a steady state solution $V_{\epsilon}=V_{0}+\epsilon V_{1}$ of a Hamiltonian system with a Hamiltonian $H_{\epsilon}=H_{0}+\epsilon H_{1}$ having an unstable manifold.

We remark that any velocity field of the form $(0, V(r), W(r))$ satisfies the Euler equations for the fluid regardless of the functions $V(r)$ and $W(r)$. The linear stability of these flows to axisymmetric perturbations was first considered by Rayleigh, who found that the flow $(0, V(r), 0)$ is stable only if

$$
\Phi=r^{-3} \frac{d}{d r}\left(r^{2} V^{2}(r)\right)>0
$$

for all $r$ in the domain of interest. Synge [1933] showed that $\Phi>0$ is necessary and sufficient for stability. Howard and Gupta [1962] derived a sufficient condition for linear stability of the flow with velocity $(0, V(r), W(r))$, including an axial velocity component $W$. The condition is

$$
J=\Phi /(d W / d r)^{2}>1 / 4 .
$$

Thus if the Richardson number $J$ exceeds $1 / 4$ everywhere in the domain of interest, then the flow is linearly stable.

As already noted the two cases $W=0$ and $W \neq 0$ possess different symmetry properties with respect to reflections $z \rightarrow-z$. The former has the symmetry $O(2) \times S O(2)$, while the latter has the symmetry $S O(2) \times S O(2)$. This distinction is important for the structure of the full problem (compare $\S 2.1$ and $\S 2.9$ ), but does not affect the linear stability calculation, i.e.,, the calculation of the quantity $p q$ that distinguishes splitting from passing. In the following we restrict ourselves to the reflection symmetric flows $(0, V(r), 0)$ satisfying the inviscid Rayleigh criterion for stability. The results of our analysis are presented for flows selected from the two-parameter family of Burger vortices given by

$$
V(r)=\frac{\Gamma}{2 \pi r}\left(1-e^{-\beta r^{2}}\right) .
$$

These profiles are of interest in the vortex breakdown problem. The results discussed below focus on the presence of splitting and hence of instability; no nonlinear computations have been carried out.

The flow configuration is shown in Figure 5 . The $\mathbf{E}$ axis is the axis of rotation for the system. The unperturbed flow velocity field is given by (6.3). In a coordinate system rotating with a constant angular velocity about $\mathbf{E}$, the inviscid Euler equations require the instantaneous velocity field to satisfy

$$
\frac{\partial \mathbf{v}}{\partial t}+\mathbf{v} \cdot \nabla \mathbf{v}+2 \epsilon \mathbf{E} \times \mathbf{v}=-\nabla \pi
$$


where $\mathbf{E}=(\cos \phi,-\sin \phi, 0)$. Here $\epsilon$ is the strength of the external Coriolis force. For $\epsilon=0$ we find that any flow $(0, V(r), 0)$ satisfies $(6.4)$. In the presence of the external Coriolis force the following is an exact solution of the inviscid Euler equations (6.4):

$$
\mathbf{u}_{0}=(0, V(r),-2 \epsilon r \sin \phi), \quad \pi_{0}=\int V^{2} / r d r-2 \epsilon^{2} r^{2} \sin ^{2} \phi .
$$

We now consider the linear stability analysis of this flow. The linearized equations for the disturbance field $(u, v, w, p)$ are

$$
\begin{aligned}
\frac{\partial u}{\partial t}+\frac{V}{r} \frac{\partial u}{\partial \phi}-2 \frac{V}{r} v-2 \epsilon r \sin \phi \frac{\partial u}{\partial z}-2 \epsilon w \sin \phi & =-\frac{\partial p}{\partial r} \\
\frac{\partial v}{\partial t}+\frac{V}{r} \frac{\partial v}{\partial \phi}+\left(\frac{V}{r}+V^{\prime}(r)\right) u-2 \epsilon r \sin \phi \frac{\partial v}{\partial z}-2 \epsilon w \cos \phi & =-\frac{1}{r} \frac{\partial p}{\partial \phi} \\
\frac{\partial w}{\partial t}+\frac{V}{r} \frac{\partial w}{\partial \phi}-2 \epsilon r \sin \phi \frac{\partial w}{\partial z} & =-\frac{\partial p}{\partial z} \\
\frac{1}{r} \frac{\partial}{\partial r}(r u)+\frac{1}{r} \frac{\partial v}{\partial \phi}+\frac{\partial w}{\partial z} & =0 .
\end{aligned}
$$

Dispersion curves for the Burger's vortex (6.3) with $\frac{\Gamma}{2 \pi}=1$ and $\beta=1$ are shown in Figure 6 . The solid and dashed curves correspond to axisymmetric $(m=0)$ and helical $(m=1)$ modes. For $\epsilon=0$ the system (6.6) has invariant subspaces characterized by different azimuthal wavenumbers. Perturbations with $\epsilon \neq 0$ couple these subspaces. As a result the movement of the eigenvalues changes from passing to splitting provided the azimuthal wavenumbers differ by 1 (Figure 7). We can associate vortex instabilities with the degeneracies (crossing points in Figure 6) caused by two physically distinguishable eigenmodes of the unperturbed vortex having the same eigenfrequency.

\section{Discussion}

In many problems stability results can be obtained from variational principles using the available conserved quantities (momentum maps or Casimir functions). As in the example of columnar flows above such a variational formulation exists for many exact solutions in two-dimensional hydrodynamics and plasma physics, as well as for more general Hamiltonian systems, and allows one to use the conserved quantities to establish the nonlinear stability of such equilibria. The situation is less clear in three-dimensional hydrodynamics. In some cases, however, it is possible to use the conserved quantities obtained via Noether's theorem to prove that an equilibrium must be linearly unstable. If the system is distorted (perturbed) in such a way that at least one conserved quantity is lost, its evolution satisfies equations of the form

$$
\frac{d}{d t} F(u)=\epsilon G(u)
$$

where $F(u)$ is a conserved quantity (quantities) for the undistorted system (obtained via Noether's theorem) and $G(u)$ is a functional describing the rate of loss of the conserved quantity (quantities) $F(u)$. Here $\epsilon$ is the strength of the system symmetry breaking perturbation. Although equations (7.1) are fully nonlinear, their linearization about $u=0$ is given by

$$
\left\langle L_{F}, \frac{d u}{d t}\right\rangle=\epsilon\left\langle L_{G}, u\right\rangle
$$


where $L_{F}=D F(0)$ and $L_{G}=D G(0)$ are two linear operators. Equation (7.2) is valid under the assumption that $\|u\|$ is small. In the case in which the distortion leads to a parametric instability involving two critical modes $u_{1}$ and $u_{2}$,

$$
\frac{d A_{1}}{d t}=\epsilon p A_{2}, \quad \frac{d A_{2}}{d t}=\epsilon q A_{1}
$$

where $A_{1}$ and $A_{2}$ are the corresponding amplitudes. It follows that instability is present if $p q>0$, with a growth rate given by

$$
\sigma^{2}=\epsilon^{2} p q
$$

An expression for $\sigma^{2}$ can be found in terms of the critical modes and $L_{F}, L_{G}$ only. Since

$$
u=A_{1}(t) u_{1}+A_{2}(t) u_{2},
$$

it follows that

$$
p\left\langle L_{F}, u_{1}\right\rangle A_{2}+q\left\langle L_{F}, u_{2}\right\rangle A_{1}=\left\langle L_{G}, u_{1}\right\rangle A_{1}+\left\langle L_{G}, u_{2}\right\rangle A_{2},
$$

and hence that

$$
p\left\langle L_{F}, u_{1}\right\rangle=\left\langle L_{G}, u_{2}\right\rangle, \quad q\left\langle L_{F}, u_{2}\right\rangle=\left\langle L_{G}, u_{1}\right\rangle .
$$

Finally, therefore,

$$
\sigma^{2}=\epsilon^{2} \frac{\left\langle L_{G}, u_{1}\right\rangle\left\langle L_{G}, u_{2}\right\rangle}{\left\langle L_{F}, u_{1}\right\rangle\left\langle L_{F}, u_{2}\right\rangle} .
$$

If the quantity (7.8) is positive the equilibrium is linearly unstable. The formula (7.8) may be viewed as an analogue of the formula for the movement of eigenvalues in the context of dissipation induced instabilities (see Bloch et al. [1993]).

The above discussion illustrates well the basic point of this paper: that system symmetry breaking perturbations of Hamiltonian systems with symmetry can, under the appropriate circumstances, lead to the loss of stability. These instabilities take place whenever the loss of symmetry results in the splitting of double eigenvalues and are important in applications since they occur on a dynamical time scale. This is so, for example, for the elliptical instability of columnar flow (Pierrehumbert [1986], Bayly [1986]). As discussed here (see also Guckenheimer and Mahalov [1992]) the origin of this instability is universal. It requires only the presence of reflection symmetry in the axial direction. If the two modes are interchanged by this symmetry, then the coefficients of the $S O(2)$-breaking terms must be equal i.e., $p=q$ in (3.2), and so $p q>0$ implying splitting. This argument establishes the existence of an instability without the necessity of having to carry out even the linear stability calculation. In this paper we have extended this approach to other multiple eigenvalues, and in particular considered the case of the Hamiltonian Hopf bifurcation, with or without an additional (axial) reflection symmetry. In this case when the system symmetry is broken, the eigenvalues may either split or bounce, indicating the need for a linear stability analysis. In addition we have shown how the symmetries of the system can be used to write down the truncated normal forms describing the growth and saturation of these instabilities. We have described the Hamiltonian structure of the resulting normal forms, and showed that in the simplest cases of interest these normal forms are completely integrable. As a result a complete description of the local dynamics becomes possible.

We focused on systems with the symmetries $S O(2) \times S O(2)$ or $O(2) \times S O(2)$, where the first group refers to periodic boundary conditions in the axial direction and the second to rotational invariance. For such systems instabilities of the type discussed here are expected to be always present since 
the translational invariance in the axial direction implies that the axial wavenumber of the modes is available as a parameter that can be tuned to force the coalescence of dangerous eigenmodes on the imaginary axis. The dangerous interactions are precisely those for which independent passing does take place in the unperturbed problem (cf. Dellnitz et al. [1992]). As illustrated in Figure 8, once the azimuthal $S O(2)$ symmetry is reduced by the system symmetry breaking perturbation the bifurcations that take place as a function of the detuning $\lambda$ are now non-semisimple double Hopf bifurcations with $1: 1$ resonance (cf. van Gils et al. [1990]).

Acknowledgements We thank Mary Silber and Vivien Kirk for helpful discussions on the Hamiltonian structure of normal forms.

\section{Bibliography}

D. Armbruster and A. Mahalov [1992], On the explicit symmetry breaking in the Taylor-Couette problem, Phys. Lett. A, 167, 251-254.

B.J. Bayly [1986], Three-dimensional instability of elliptical flow, Phys. Rev. Lett., 57, 2160-2163.

T.B. Benjamin [1967], Instability of periodic wavetrains in nonlinear dispersive systems, Proc. Roy. Soc. London A, 299, 59-75.

A.M. Bloch, P.S. Krishnaprasad, J.E. Marsden and T.S. Ratiu [1993], Dissipation-induced instabilities, preprint.

J.D. Crawford [1992], Normal forms for driven surface waves: boundary conditions, symmetry and genericity, Physica D, 52, 429-457.

R. Cushman and D.L. Rod [1982], Reduction of the semisimple 1: 1 resonance, Physica D, 6, $105-112$.

planar vector fields,

G. Dangelmayr and E. Knobloch [1991], Hopf bifurcation with broken circular symmetry, Nonlinearity, 4, 399-427.

M. Dellnitz, I. Melbourne and J.E. Marsden [1992], Generic bifurcation of Hamiltonian vector fields with symmetry, Nonlinearity, 5, 979-996.

Z.C. Feng and P.R. Sethna [1989], Symmetry breaking bifurcations in resonant surface waves, J. Fluid Mech., 199, 495-518.

Z.C. Feng and P.R. Sethna [1990], Global bifurcations and chaos in parametrically forced systems with one-one resonance, Dyn. Stab. Systems, 5, 201-225.

Z.C. Feng and S. Wiggins [1993], On the existence of chaos in a class of two-degree-of-freedom, damped, strongly parametrically forced mechanical systems with broken $O(2)$ symmetry, $Z$. angew. Math. Phys., 44, 201-248.

D.M. Galin [1982], Versal deformations of linear Hamiltonian systems, AMS Transl. 118, 1-12, (Trudy Sem. Petrovsk. 1, 63-74, 1975).

S.A. van Gils, M. Krupa and W.F. Langford [1990], Hopf bifurcation with non-semisimple $1: 1$ resonance, Nonlinearity, 3, 825-850. 
J. Guckenheimer and A. Mahalov [1992], Instability induced by symmetry reduction, Phys. Rev. Lett., 68, 2257-2260.

G. Haller and S. Wiggins [1992], Geometry and chaos near resonant equilibria of three degree of freedom Hamiltonian systems, preprint.

G. Haller and S. Wiggins [1993], Orbits homoclinic to resonances: the Hamiltonian case, Physica $D, 66,298-346$.

L.N. Howard and A.S. Gupta [1962], On the hydrodynamic and hydromagnetic stability of swirling flows, J. Fluid Mech., 14, 463-476.

T. Iwai [1985], On reduction of two degrees of freedom Hamiltonian systems by an $S^{1}$ action, and $S O_{0}[1,2]$ as a dynamical group, J. Math. Phys., 26, 885-893.

M.F. Jorgensen and P.L. Christiansen [1992], Hamiltonian structure for a modified discrete selftrapping dimer, preprint.

R.R. Kerswell [1992], Instabilities of tidally and precesionally induced flows, preprint.

E. Knobloch [1992a], Nonlocal amplitude equations, in Pattern Formation in Complex Dissipative Systems, S. Kai, ed., World Scientific, 263-274.

E. Knobloch [1992b], Bifurcations in rotating systems, in Theory of Solar and Planetary Dynamos: Introductory Lectures, M.R.E. Proctor and A.D. Gilbert, eds., Cambridge University Press, in press.

E. Knobloch and M. Silber [1993], Oscillatory convection in a rotating layer, Physica D, 63, 213232.

M.G. Krein [1950], A generalization of several investigations of A.M. Liapunov on linear differential equations with periodic coefficients, Dokl. Akad. Nauk SSSR, 73, 445-448.

M. Kummer [1979] On resonant Hamiltonian systems with finitely many degrees of freedom, Springer Lecture Notes in Physics 93, 57-75.

M. Kummer [1990], On resonant classical Hamiltonians with $n$ frequencies, J. Diff. Eq., 83, 220243.

S. Leibovich, S.N. Brown and Y. Patel [1986], Bending waves on inviscid columnar vortices, J. Fluid Mech., 173, 595-624.

D. Lewis and J.E. Marsden [1989] The Hamiltonian-dissipative decomposition of normal forms of vector fields. Proc of the conf. on Bifurcation Theory and its Numerical Analysis, Xi'an Jaitong Univ. Press, 51-78.

A. Mahalov [1993], The instability of rotating fluid columns subjected to a weak external Coriolis field, Phys. Fluids A, 5, 891-900.

J.E. Marsden [1992], Lectures on Mechanics, Cambridge University Press.

A. Mielke [1992] Hamiltonian and Lagrangian Flows on Center Manifolds, with Applications to Elliptic Variational Problems, Springer Lecture Notes in Mathematics 1489.

J. Miles [1991], Nonlinear asymmetric excitation of edge waves, Int. J. Appl. Math., 46, 101-108. 
S.T. Milner [1991], Square patterns and secondary instabilities in driven capillary waves, J. Fluid Mech., 225, 81-100.

W. Nagata [1988], Dynamics near a symmetric Hopf bifurcation, Proc. Roy. Soc. Edin., 108A, 249-267.

W. Nagata [1989], Nonlinear Faraday resonance in a box with a square base, J. Fluid Mech., 209, $265-284$.

R.T. Pierrehumbert [1986], Universal short-wave instability of 2-dimensional eddies in an inviscid fluid, Phys. Rev. Lett., 57, 2157-5159.

H. Riecke, J.D. Crawford and E. Knobloch [1988], Time-modulated oscillatory convection, Phys. Rev. Lett., 61, 1942-1945.

M. Silber and E. Knobloch [1991], Hopf bifurcation on a square lattice, Nonlinearity, 4, 1063-1106.

J.W Swift [1988], Hopf bifurcation with the symmetry of a square, Nonlinearity, 1, 333-377.

J.L. Synge [1933], The instability of heterogeneous fluids, Trans. R. Soc. Can., 27, 1-18.

A. Szeri and P. Holmes [1988], Nonlinear stability of axisymmetric swirling flows, Phil. Trans. R. Soc. Lond. A, 326, 327-354.

V.A. Vladimirov and K. Il'in [1988], Three-dimensional instability of an elliptical Kirchoff vortex, Fluid Dynamics, 3, 356-360.

F.A. Waleffe [1990], On the three-dimensional instability of a strained vortex, Phys. Fluids A, 2, $76-80$.

D. Walgraef [1988], External forcing of spatio-temporal patterns, Europhys. Lett., 7, 485-491.

\section{Figure Captions}

Figure 1. Bifurcation diagram for $m=1$ in the + case (splitting): (a) $c>1$, (b) $c<1$. The label $S$ denotes a stable equilibrium (center) while $U$ denotes an unstable equilibrium (saddle).

Figure 2. Bifurcation diagram for $m=1$ in the - case (passing). The label $S$ denotes a stable equilibrium (center) while $U$ denotes an unstable equilibrium (saddle).

Figure 3. The transition between splitting and passing for $m=1$, showing (a) $N(\lambda)$ and (b) $w(\lambda)$ when $q=0$. This case connects Figure $1 \mathrm{~b}$ with Figure 2 .

Figure 4. Elliptical instability $(m=2)$. Phase portrait for standing oscillations (a) $\lambda>\epsilon>0$, (b) $|\lambda|<\epsilon,(\mathrm{c}) \lambda<-\epsilon$.

Figure 5. Coordinate system for the columnar vortex subjected to an external Coriolis force.

Figure 6. Dispersion curves for the Burger's vortex. The solid and dashed curves correspond to axisymmetric $(m=0)$ and helical $(m=1)$ modes.

Figure 7. Movement of eigenvalues: (a) independent passing $(\epsilon=0)$, (b) splitting $(\epsilon \neq 0)$.

Figure 8. Independent passing $(\epsilon=0)$ and non-semisimple Hamiltonian Hopf $(\epsilon \neq 0)$. 